

\title{
Helmholtz and Gibbs ensembles, thermodynamic limit and bistability in polymer lattice models \\ Stefano Giordano
}

\section{To cite this version:}

Stefano Giordano. Helmholtz and Gibbs ensembles, thermodynamic limit and bistability in polymer lattice models. Continuum Mechanics and Thermodynamics, 2018, 30 (3), pp.459-483. 10.1007/s00161-017-0615-5 . hal-02337983

\section{HAL Id: hal-02337983 \\ https://hal.science/hal-02337983}

Submitted on 24 Sep 2020

HAL is a multi-disciplinary open access archive for the deposit and dissemination of scientific research documents, whether they are published or not. The documents may come from teaching and research institutions in France or abroad, or from public or private research centers.
L'archive ouverte pluridisciplinaire HAL, est destinée au dépôt et à la diffusion de documents scientifiques de niveau recherche, publiés ou non, émanant des établissements d'enseignement et de recherche français ou étrangers, des laboratoires publics ou privés. 


\title{
Helmholtz and Gibbs ensembles, thermodynamic limit and bistability in polymer lattice models
}

Received: date / Accepted: date

\begin{abstract}
Representing polymers by random walks on a lattice is a fruitful approach largely exploited to study configurational statistics of polymer chains and to develop efficient Monte Carlo algorithms. Nevertheless, the stretching and the folding/unfolding of polymer chains within the Gibbs (isotensional) and the Helmholtz (isometric) ensembles of the statistical mechanics has not been yet thoroughly analysed by means of the lattice methodology. This topic, motivated by the recent introduction of several single-molecule force spectroscopy techniques, is investigated in the present paper. In particular, we analyse the force-extension curves under the Gibbs and Helmholtz conditions and we give a proof of the ensembles equivalence in the thermodynamic limit for polymers represented by a standard random walk on a lattice. Then, we generalize these concepts for lattice polymers that can undergo conformational transitions or, equivalently, for chains composed of bistable or two-state elements (that can be either folded or unfolded). In this case, the isotensional condition leads to a plateau-like forceextension response, whereas the isometric condition causes a sawtooth-like force-extension curve, as predicted by numerous experiments. The equivalence of the ensembles is finally proved also for lattice polymer systems exhibiting conformational transitions.
\end{abstract}

Keywords Polymer lattice models · Polymer stretching · Isotensional and isometric ensembles · Ensemble equivalence · Lattice Green functions

\section{Introduction}

Several modelling approaches have been introduced to describe the physics of macromolecules. Concisely, such models can be classified as off-lattice polymer models [1,2] and lattice polymer models [3, 4]. While off-lattice models refer to all the continuous models represented by arbitrary interaction potentials between the monomers or elements of the chain, lattice models consider the chains embedded in regular lattices and can be viewed as coarse-grained versions of continuous polymer models. Typically, off-lattice models have been introduced to approach theoretical problems [5,6], whereas lattice methodologies are more indicated for simulations $[7,8]$.

An important example of lattice model implementation is represented by the so-called directed walks [3]. They are defined as random walks making only steps that have non-negative projection on a given preferential direction. For example, they are useful to model polymers immersed in a flow or charged polymers exposed to an electric field. A relevant generalization concerns the directed walks

Stefano Giordano

Institute of Electronics, Microelectronics and Nanotechnology - UMR 8520, LIA LICS, Univ. Lille, CNRS, Centrale Lille, ISEN, Univ. Valenciennes, F-59000 Lille, France.

Tel.: +33 (0)3 20197958

Fax: +33 (0)3201979 84

ORCID: 0000-0003-4023-5384

E-mail: stefano.giordano@iemn.univ-lille1.fr 


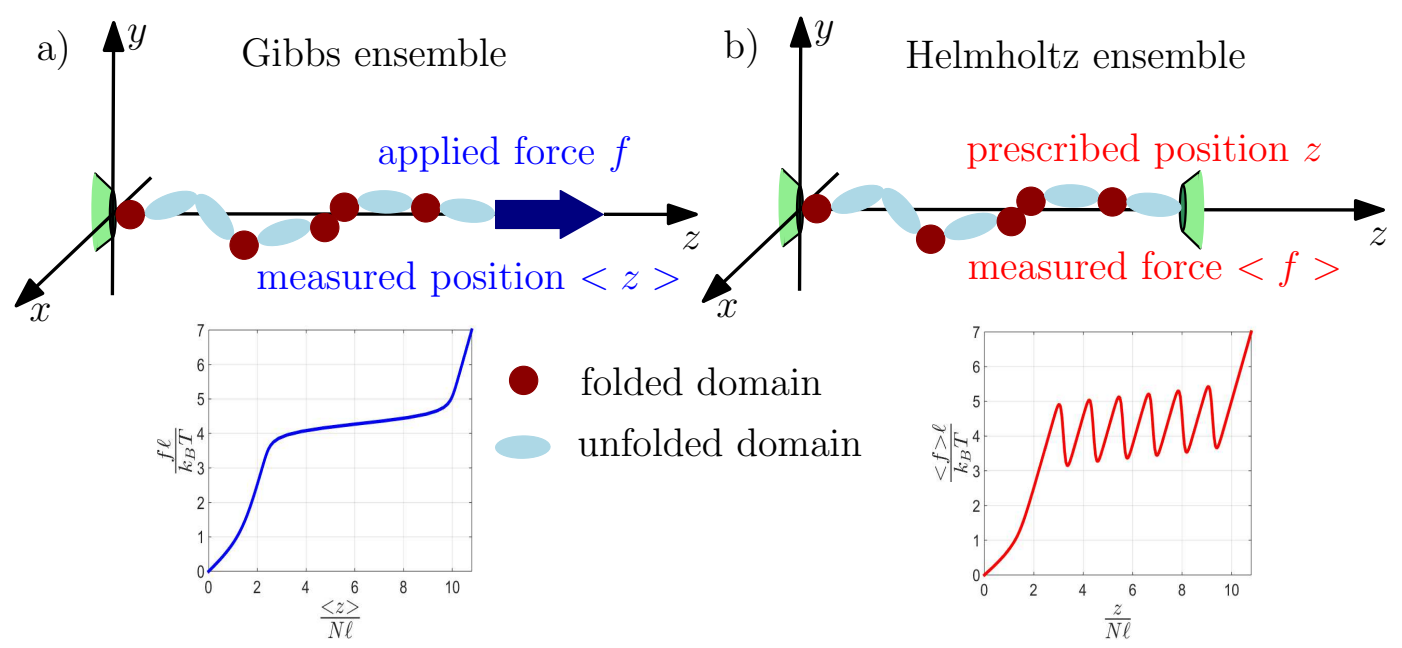

Fig. 1 Single-molecule stretching experiments conducted within the Gibbs (a) and the Helmholtz (b) ensembles. Each domain of the chain may experience a conformational transition between folded (length $\ell$ ) and unfolded (length $\chi \ell$ ) states. The measured force-extension curve under isotensional Gibbs conditions exhibits a force plateau describing the simultaneous unfolding of the domains. On the other hand, the isometric Helmholtz conditions produce a sawtooth-like response characterized by a series of force peaks corresponding to the sequential unfolding of domains.

in random environment, useful to simulate real polymers in random media and to develop several applications in general statistical mechanics [9].

A second example of lattice systems is given by the self avoiding walks. They represent polymers immersed in a solvent. Here the monomers of the chain are favourably surrounded by molecules of the solvent, thus creating the so-called excluded volume phenomenon [10]. Remarkably, these models have a formal relation with the physics of critical phenomena. Indeed, self avoiding walks exhibit the same properties of a magnetic system near its critical point [11].

In recent years, the mechanical response of single polymer chains or macromolecules of biological origin has been extensively examined through atomic-force microscopes, laser optical tweezers, magnetic tweezers, and other micro-electro mechanical systems, which are able to stretch individual molecules by probing forces and extensions [12-14]. The direct quantification of the elasticity of single molecules (force spectroscopy) allowed to investigate for the first time the themodynamics and the statistical mechanics of small systems $[15,16]$. Such experimental activities have encouraged the improvement of theoretical approaches to describe the thermoelastic properties of polymer chains. Classical examples of off-lattice models are given by the freely-jointed chain model $[1,17]$, the worm-like chain model [18, 19] and several advanced generalizations [20-25]. The utilization of lattice polymer models for studying the chain stretching configuration is the subject of the present work, where we consider both the Gibbs and the Helmholtz ensembles of the statistical mechanics.

As a matter of fact, the stretching of a polymer chain can be performed within two distinct statistical ensembles and the different thermoelastic responses can be easily observable at both theoretical and experimental level. The first ensemble is the so-called Gibbs (or isotensional) ensemble, characterized by a deterministic force applied to the free end of the chain, being the other end clamped at the origin of the axes. The second one is the Helmholtz (or isometric) ensemble, obtained with both the ends of the polymer tethered at two different points of the space. Because of the consideration of these two dual boundary conditions, one has to introduce the issue of the equivalence of statistical ensembles. It means that, when the thermodynamic limit is not satisfied (small number $m$ of monomers), the two ensembles are not equivalent, leading to different force-extension curves [20,21]. On the other hand, whenever $m$ approaches infinity, it is important to elucidate if the equivalence of ensembles occurs or not. Several results confirm the equivalence of the ensembles in the thermodynamic limit for off-lattice or continuous polymer systems $[26,27]$. Here, we will prove that this property holds on also for lattice polymer models.

In many cases of practical interest, the macromolecule stretching leads to the process of unfolding, corresponding to the conformational transition of the domains from the folded state to the unfolded 
one $[28,29]$. It means that each domain can switch its state between two metastable chemical configurations. This point can be described by a potential energy function composed of two minima, thus exhibiting a mechanical bistability. The measure of the force-extension curve with different devices can situate the experiment either within the Gibbs (soft device) or the Helmholtz (hard device) ensemble. Typical measured force-extension curves are shown in Fig.1 for the two ensembles. The Gibbs response exhibits a plateau-like shape and can be interpreted by supposing that the conformational change occurs simultaneously for all the domains at a given threshold force (cooperative process) $[28,29]$. On the other hand, the Helmholtz response shows a sawtooth-like curve, proving that the domains unfold sequentially in reaction to the increasing extension (non-cooperative process) [28,29]. This complex scenario, already investigated and confirmed through experiments [30,31] and theories [32-34] for offlattice models, is studied in the present paper by means of lattice models. In particular, we obtain the mathematical form of the force-extension curves in both ensembles, we prove the qualitative agreement with the responses shown in Fig.1, and finally, we rigorously demonstrate the ensembles equivalence in the thermodynamic limit.

A relevant dissimilarity between the Gibbs and Helmholtz needs to be discussed in more detail. Under isotensional conditions the elements of the chain behave independently, thus generating a partition function that is a power of a given quantity, being the exponent equal to the total number $m$ of monomers. On the other hand, the partition function under isometric conditions cannot be written in a $m$-th power form since the peculiar boundary conditions imposed generate an interdependence among the lengths of elements, ultimately representing an effective interaction. It is important to stress that this interaction is not a real physical interaction among neighboring elements, but rather a resulting interaction induced by the isometric prescription. It is a typical effect of the statistical mechanics of small systems. From the mathematical point of view, this point can be observed in the large complexity of the Helmholtz partition function calculation with respect the the Gibbs one. For continuous or off-lattice polymer models this issue is typically dealt with by means of the Laplace transform relationship existing between the Gibbs and Helmholtz partition functions [34]. Here, differently, the Helmholtz partition function has been obtained through a lattice Green function, which allows for the path counting on the lattice. Also for the case with conformational transitions, an $a d$ hoc generalized lattice Green function has been introduced to identify the Helmholtz partition function. Being, in general, the lattice Green function written in the form of a multiple integral, the analysis of the ensembles equivalence has been approached by a suitable asymptotic technique, namely the multivariate saddle point method. This approach permitted the analysis of the thermodynamic limit for both systems without and with conformational transitions.

It is important to remark that the conformational transitions introduced here to model the folding/unfolding processes in macromolecules are also able to describe the discrete phase transformations observed in several biological and non-biological systems such as macromolecular hairpins, adhesive clusters, ferromagnetic alloys, indented substrates and plastic materials $[35,36]$.

The structure of the paper is the following. In Section 2, we review the problem of the path counting on a $d$-dimensional lattice, useful for the successive developments. In Sections 3 and 4 we introduce the Gibbs and the Helmholtz ensembles, respectively. Then, in Section 5, we prove the ensemble equivalence for long chains by means of an ad hoc application of the multivariate saddle point method. To conclude, in Section 6, we generalize previous concepts to the case of lattice polymers undergoing conformational transitions, i.e. characterized by two-state or bistable elements. In this case, both statistical ensembles and their equivalence are relevant for the interpretation of several force spectroscopy experiments, as thoroughly discussed herein. Finally, three appendices contain some detailed mathematical developments.

\section{Path counting on the $\mathbb{Z}^{n}$ lattice}

We take into consideration a regular $\mathbb{Z}^{n}$ lattice and we consider the analogy between random paths and polymer chains. Based on this formal analogy, we develop our discussion using both the terminology of random paths and of polymer chains. The position within the lattice is specified by the vector $\mathbf{x} \in \mathbb{Z}^{n}$. We consider a random path starting at the origin of the axes and we define $P_{m}(\mathbf{x})$ as the probability to arrive in $\mathbf{x}$ at the step (or time) $m$. In order to obtain a general equation governing the dynamics of $P_{m}(\mathbf{x})$, we use the law of total probability in the form $P(B)=\sum_{j=1}^{N} P\left(B \mid A_{i}\right) P\left(A_{i}\right)$, where $B$ is an 
event $B \subset \Omega$ (where $\Omega$ is the sample space) and $\left\{A_{i}, i=1, \ldots, N\right\}$ is a partition of $\Omega$ [37]. Moreover, $P\left(B \mid A_{i}\right)$ are the conditional probabilities. We identify the event $B$ with the condition of being in $\mathrm{x}$ at time $m+1$. Moreover, we identify the events $A_{i}$ with the condition of being in a given lattice position at time $m$. Finally, $P\left(B \mid A_{i}\right)$ is equal to $1 /(2 n)$ (the reciprocal of number of directions in $\mathbb{Z}^{n}$ ) for isotropic processes if the position of the site defining $A_{i}$ is adjacent to $\mathbf{x}$ and equal to zero for non-neighbouring sites. The above introduced law of total probability immediately leads to

$$
P_{m+1}(\mathbf{x})=\frac{1}{2 n} \sum_{i=1}^{n}\left[P_{m}\left(\mathbf{x}-\mathbf{e}_{i}\right)+P_{m}\left(\mathbf{x}+\mathbf{e}_{i}\right)\right]
$$

where the vectors $\mathbf{e}_{i}$ represent a canonical base in $\mathbb{Z}^{n}$. This evolution equation can be solved by imposing an arbitrary initial condition $P_{0}(\mathbf{x})$. Often, we will adopt the condition $P_{0}(\mathbf{x})=\delta(\mathbf{x})$ (discrete Delta function) if we start the random path at the origin of the axes or, equivalently, if the polymer is tethered at $\mathbf{x}=\mathbf{0}$. We define the generating function $\mathcal{P}(\mathbf{x}, z)$ as follows

$$
\mathcal{P}(\mathbf{x}, z)=\sum_{m=0}^{+\infty} P_{m}(\mathbf{x}) z^{-m}
$$

where $\mathbf{x} \in \mathbb{Z}^{n}$ and $z \in \mathbb{C}$. It can be also view as the $z$-transform of the sequence $P_{m}$, where $\mathbf{x}$ is considered as a parameter [38]. By applying Eq.(2) to Eq.(1), we easily develop an equation for the generating function

$$
z \mathcal{P}(\mathbf{x}, z)-\sum_{i=1}^{n} \frac{1}{2 n}\left[\mathcal{P}\left(\mathbf{x}-\mathbf{e}_{i}, z\right)+\mathcal{P}\left(\mathbf{x}+\mathbf{e}_{i}, z\right)\right]=z P_{0}(\mathbf{x}),
$$

where, as above said, we will consider $P_{0}(\mathbf{x})=\delta(\mathbf{x})$. In order to obtain an explicit solution of Eq.(3), we suppose to use the numbers $\mathcal{P}(\mathbf{x}, z) \forall \mathbf{x} \in \mathbb{Z}^{n}$ as the Fourier coefficients of a periodic function $f$ defined in $[-\pi, \pi]^{n} \subset \Re^{n}$. Hence, we define $f(\mathbf{k}, z)=\sum_{\mathbf{x} \in \mathbb{Z}^{n}} \mathcal{P}(\mathbf{x}, z) \mathrm{e}^{-i \mathbf{k} \cdot \mathbf{x}}$, with $\mathbf{k} \in[-\pi, \pi]^{n} \subset \Re^{n}$. So, we have the Fourier coefficients defined through the classical expression

$$
\mathcal{P}(\mathbf{x}, z)=\frac{1}{(2 \pi)^{n}} \int_{-\pi}^{+\pi} \ldots \int_{-\pi}^{+\pi} f(\mathbf{k}, z) \mathrm{e}^{i \mathbf{k} \cdot \mathbf{x}} d \mathbf{k} .
$$

If we now apply the operator $\sum_{\mathbf{x} \in \mathbb{Z}^{n}} \mathrm{e}^{-i \mathbf{k} \cdot \mathbf{x}}$ to the shifted terms of Eq.(3), we obtain $\sum_{\mathbf{x} \in \mathbb{Z}^{n}} \mathcal{P}(\mathbf{x} \pm$ $\left.\mathbf{e}_{i}, z\right) \mathrm{e}^{-i \mathbf{k} \cdot \mathbf{x}}=\mathrm{e}^{ \pm i \mathbf{k} \cdot \mathbf{e}_{i}} f(\mathbf{k}, z)$. Thus, from Eq.(3) with $P_{0}(\mathbf{x})=\delta(\mathbf{x})$, we finally get the periodic function as $f(\mathbf{k}, z)=1 /\left(1-\frac{1}{n z} \sum_{i=1}^{n} \cos k_{i}\right)$, and the solution of Eq.(3) reads

$$
\mathcal{P}(\mathbf{x}, z)=\frac{1}{(2 \pi)^{n}} \int_{-\pi}^{+\pi} \ldots \int_{-\pi}^{+\pi} \frac{\mathrm{e}^{i \mathbf{k} \cdot \mathbf{x}} d \mathbf{k}}{1-\frac{1}{n z} \sum_{i=1}^{n} \cos k_{i}} .
$$

The previous expression is related to the so-called lattice Green function, which is of frequent occurrence in many lattice statistical problems with isotropic or anisotropic nearest-neighbour interactions [3941]. This formalism is also largely used to model disordered resistors networks, which represent a very useful tool to model transport phenomena in heterogeneous or composite physical systems [42-45].

Now, since $\mathcal{P}(\mathbf{x}, z)=\sum_{m=0}^{+\infty} P_{m}(\mathbf{x}) z^{-m}$, we explicitly determine $P_{m}(\mathbf{x})$ by remembering that for $|z|>a$ the following relation [38]

$$
\frac{1}{1-a z^{-1}}=\sum_{m=0}^{+\infty} a^{m} z^{-m} \quad(|z|>a)
$$

holds true. It represents the $z$-transform of the sequence $a^{m} \forall m \in \mathbb{N}$ [38]. Indeed, the application of this property to Eq.(5) yields the result

$$
P_{m}(\mathbf{x})=\frac{1}{(2 \pi)^{n}} \int_{-\pi}^{+\pi} \ldots \int_{-\pi}^{+\pi} \mathrm{e}^{i \mathbf{k} \cdot \mathbf{x}}\left[\frac{1}{n} \sum_{i=1}^{n} \cos k_{i}\right]^{m} d \mathbf{k} .
$$


This is the probability of finding the random path starting from $\mathbf{0}$ at the position $\mathbf{x}$ after $m$ steps. For the following purposes, it is important to remark that this probability is equal to the ratio between the number of paths from $\mathbf{0}$ to $\mathbf{x}$ and the total number of paths starting from $\mathbf{0}$ and arriving at any point (both composed of $m$ steps). This property simply derives from the classical definition of probability [37]. Since the total number of paths starting from $\mathbf{0}$ and composed of $m$ steps is given by $(2 n)^{m}$ (if we work in $\mathbb{Z}^{n}$ ), we can easily obtain the number of paths from $\mathbf{0}$ to $\mathbf{x}$ with $m$ steps. We name this number Helmholtz partition function and we have

$$
Z_{H}(\mathbf{x})=(2 n)^{m} P_{m}(\mathbf{x})=\frac{2^{m-n}}{\pi^{n}} \int_{-\pi}^{+\pi} \cdots \int_{-\pi}^{+\pi} \mathrm{e}^{i \mathbf{k} \cdot \mathbf{x}}\left[\sum_{i=1}^{n} \cos k_{i}\right]^{m} d \mathbf{k} .
$$

The origin of this terminology will be elucidated in a following Section.

Finally, we have obtained the number of paths on an $n$-dimensional lattice connecting $\mathbf{0}$ to $\mathbf{x}$ with $m$ steps. In other words, we have solved the problem of counting the number of paths or polymer configurations connecting two points in $\mathbb{Z}^{n}$, having a fixed number $m$ of segments.

\section{The Gibbs ensemble: isotensional conditions}

We consider a polymer with $m$ segments randomly arranged on the $\mathbb{Z}^{n}$ lattice, each of length $\ell$. We suppose to apply a force $\mathbf{f}$ to the last element of the chain when the first element is tethered at the origin of the axes. The positions of the elements are $\mathbf{x}_{1}, \ldots, \mathbf{x}_{m}$ and the Hamiltonian of the system is simply given by the potential energy of the applied force

$$
\mathcal{H}=-\mathbf{f} \cdot \mathbf{x}_{m}
$$

Each segment of the lattice polymer is represented by a vector $\ell \boldsymbol{\sigma}_{i} \forall i=1, \ldots, m$, the vectors $\boldsymbol{\sigma}_{i}$ assuming their values from the set $\Theta=\left\{ \pm \mathbf{e}_{k}, k=1, \ldots, n\right\}$, which represents the standard base in $\mathbb{Z}^{n}$, extended to both positive and negative elements. Besides, $\Sigma$ is the sequence $\left(\boldsymbol{\sigma}_{1}, \ldots, \boldsymbol{\sigma}_{m}\right)$, characterizing the actual configuration of the polymer. Of course, we have $\mathbf{x}_{m}=\ell \sum_{i=1}^{m} \boldsymbol{\sigma}_{i}$. The canonical distribution within the Gibbs ensemble is then given by

$$
P_{G}(\Sigma)=\frac{1}{Z_{G}(\mathbf{f})} \exp \left(\frac{\ell}{k_{B} T} \mathbf{f} \cdot \sum_{i=1}^{m} \boldsymbol{\sigma}_{i}\right)
$$

where the partition function $Z_{G}$ can be defined as follows

$$
Z_{G}(\mathbf{f})=\sum_{\Sigma \in \Omega} \exp \left(\frac{\ell}{k_{B} T} \mathbf{f} \cdot \sum_{i=1}^{m} \boldsymbol{\sigma}_{i}\right) .
$$

Here, $\Omega=\left\{\Sigma: \Sigma=\left(\boldsymbol{\sigma}_{1}, \ldots, \boldsymbol{\sigma}_{m}\right), \boldsymbol{\sigma}_{i} \in \Theta \forall i\right\}$ is the sample space, i.e. the set of all the possible polymer configurations. The calculation of $Z_{G}$ follows

$$
\begin{aligned}
Z_{G}(\mathbf{f}) & =\sum_{\boldsymbol{\sigma}_{1} \in \Theta} \ldots \sum_{\boldsymbol{\sigma}_{m} \in \Theta} \exp \left(\frac{\ell}{k_{B} T} \mathbf{f} \cdot \boldsymbol{\sigma}_{1}\right) \times \ldots \times \exp \left(\frac{\ell}{k_{B} T} \mathbf{f} \cdot \boldsymbol{\sigma}_{m}\right) \\
& =\left[\sum_{\boldsymbol{\sigma} \in \Theta} \exp \left(\frac{\ell}{k_{B} T} \mathbf{f} \cdot \boldsymbol{\sigma}\right)\right]^{m}=2^{m}\left[\sum_{i=1}^{n} \cosh \left(\frac{\ell f_{i}}{k_{B} T}\right)\right]^{m} .
\end{aligned}
$$

It is important to remark that within the Gibbs ensemble the elements of the chain do not interact and this point leads to a partition function which is in the form of a power with exponent $m$. The partition function allows the determination of the constitutive equation of the system through the relation

$$
\left\langle\mathbf{x}_{m}\right\rangle=k_{B} T \frac{\partial}{\partial \mathbf{f}} \log Z_{G}(\mathbf{f})=k_{B} T \sum_{i=1}^{n} \frac{\partial}{\partial f_{i}} \log Z_{G}(\mathbf{f}) \mathbf{e}_{i}
$$


which is largely used in the classical theory of polymer stretching $[1,2,20,22]$. The explicit calculation simply gives

$$
\left\langle\mathbf{x}_{m}\right\rangle=m \ell \frac{\sum_{i=1}^{n} \sinh \left(\frac{\ell f_{i}}{k_{B} T}\right) \mathbf{e}_{i}}{\sum_{i=1}^{n} \cosh \left(\frac{\ell f_{i}}{k_{B} T}\right)} .
$$

This is the force-extension relation within the Gibbs ensemble or, equivalently, under isotensional conditions.

We remark that the obtained constitutive equation is anisotropic, which means that the average value of the free end-terminal vector position is not parallel to the applied force, unless the force is applied along one axis of the reference frame. This represents an effect introduced by the polymer lattice systems, which must be carefully considered when we use these models to approximate real continuous polymer structures [46]. The comparison between continuous and lattice models for polymers has been widely investigated in literature $[47,48]$.

\section{The Helmholtz ensemble: isometric conditions}

We now consider a chain of segments with fixed extremities at $\mathbf{0}$ and $\mathbf{x}$ (isometric conditions) on the $\mathbb{Z}^{n}$ lattice. Once fixed these two boundary conditions, the system is completely free to fluctuate. Since the potential energy of the system is zero, the partition function is given by Eq.(8) and it represents the number of possible paths connecting $\mathbf{0}$ to $\mathbf{x}$ on the $\mathbb{Z}^{n}$ lattice. We remark that $\mathbf{x} \in \mathbb{Z}^{n}$ and, therefore, the actual physical position of the second end-terminal is given by $\mathbf{x}_{m}=\ell \mathbf{x}$. The canonical distribution within the Helmholtz ensemble is therefore given by

$$
P_{H}(\Sigma)=\frac{1}{Z_{H}(\mathbf{x})}
$$

where $\Sigma \in \Omega_{0}=\left\{\Sigma=\left(\boldsymbol{\sigma}_{1}, \ldots, \boldsymbol{\sigma}_{m}\right) \in \Omega: \sum_{i=1}^{m} \boldsymbol{\sigma}_{i}=\mathbf{x}\right\}$. It means that we have a uniform distribution of probability among all paths connecting the two fixed extremities. Therefore, we can also observe that $Z_{H}(\mathbf{x})$ is simply the number of elements of the set $\Omega_{0}$. So, we remark that the path counting on the $\mathbb{Z}^{n}$ lattice is of central importance to study polymers within the Helmholtz ensemble. It is also interesting to note that the partition function obtained in Eq.(8) can not be written as a power with exponent $m$. It means that within the Helmholtz ensemble there is an effective interaction among the elements. The origin of this interaction is not explicitly defined in the potential energy of the system (as, e.g., in the classical Ising model), but comes from the specific boundary conditions characterising the Helmholtz ensemble. In the classical theory of polymer stretching, the isometric conditions lead to $[1,2,20,22]$

$$
\langle\mathbf{f}\rangle=-k_{B} T \frac{\partial}{\partial \mathbf{x}} \log Z_{H}(\mathbf{x})=-k_{B} T \sum_{i=1}^{n} \frac{\partial}{\partial x_{i}} \log Z_{H}(\mathbf{x}) \mathbf{e}_{i}
$$

which is the dual counterpart of Eq.(13). It means that the Helmholtz conditions, fixing the extremities of the chain, are at the origin of a so-called entropic force, actually applied to the physical constraints tethering the polymer. In our case, the polymer configuration is defined on a lattice and therefore $Z_{H}(\mathbf{x})$ is defined for $\mathbf{x} \in \mathbb{Z}^{n}$. Then, the derivatives in Eq.(16) must be substituted with a finite difference, as follows

$$
\langle\mathbf{f}\rangle=\frac{k_{B} T}{2 \ell} \sum_{i=1}^{n} \log \frac{Z_{H}\left(\mathbf{x}-\mathbf{e}_{i}\right)}{Z_{H}\left(\mathbf{x}+\mathbf{e}_{i}\right)} \mathbf{e}_{i} .
$$

This expression represents the force-extension relation for a polymer on the lattice, where the partition function is given in Eq.(8). The choice of substituting the derivative with the finite difference is further justified below by considering the comparison of Eqs.(14) and (17) for large values of $m$, i.e. in the thermodynamic limit. 


\section{The thermodynamic limit}

Now, we want to compare the force-extension relation within the Gibbs and the Helmholtz ensembles for large values of $m$. We therefore investigate the equivalence of the ensembles in the thermodynamic limit (i.e. for $m \rightarrow \infty$ ). As usual, we say that the ensembles are equivalent if the two force-extension responses coincide in the limit of $m \rightarrow \infty$. This property has been largely discussed for continuous polymer models in recent literature and we try to extend here these results for polymers models defined on the $\mathbb{Z}^{n}$ lattice. The difficulty of this analysis resides in the fact that while the Gibbs force-extension relation is known in closed form as given in Eq.(14), its Helmholtz counterpart, stated in Eq.(17), is written in terms of the ratio of two $n$-dimensional integrals, which cannot be calculated analytically, see Eq.(8). In order to study the behavior of Eq.(17), we can write

$$
Z_{H}\left(\mathbf{x} \pm \mathbf{e}_{i}\right)=\frac{2^{m-n}}{\pi^{n}} \mathcal{Z}^{ \pm}(\mathbf{x})
$$

where we introduced the two quantities

$$
\mathcal{Z}^{ \pm}(\mathbf{x})=\int_{-\pi}^{+\pi} \ldots \int_{-\pi}^{+\pi} \exp (i \mathbf{k} \cdot \mathbf{x}) \exp \left( \pm i \mathbf{k} \cdot \mathbf{e}_{i}\right) \exp \left(m \log \sum_{i=1}^{n} \cos k_{i}\right) d \mathbf{k}
$$

Here, $\mathbf{x} \in \mathbb{Z}^{n}$ and, therefore, we have that $\mathbf{x}_{m}=\ell \mathbf{x} \in \Re^{n}$ is the real end-terminal of the chain. We define the normalized length $\mathbf{y}=\mathbf{x}_{m} /(m \ell)$ and then we simply have that $\mathbf{x}=m \mathbf{y}$. So, in terms of the normalized length, we have

$$
\mathcal{Z}^{ \pm}(\mathbf{y})=\int_{-\pi}^{+\pi} \ldots \int_{-\pi}^{+\pi} \exp \left[m\left(i \mathbf{k} \cdot \mathbf{y}+\log \sum_{i=1}^{n} \cos k_{i}\right)\right] \exp \left( \pm i \mathbf{k} \cdot \mathbf{e}_{i}\right) d \mathbf{k} .
$$

It is also useful to define a normalized force through the relation $\boldsymbol{\eta}=\frac{\ell \mathbf{f}}{k_{B} T}$. So doing, the forceextension relations can be written in terms of the normalised quantities $\mathbf{y}$ and $\boldsymbol{\eta}$. In particular, the response within the Gibbs ensemble given in Eq.(14) assumes the simpler form

$$
\langle\mathbf{y}\rangle=\frac{\sum_{i=1}^{n} \sinh \left(\eta_{i}\right) \mathbf{e}_{i}}{\sum_{i=1}^{n} \cosh \left(\eta_{i}\right)} .
$$

On the other hand, the constitutive equation within the Helmholtz ensembles given in Eq.(17) reads

$$
\langle\boldsymbol{\eta}\rangle=\frac{1}{2} \sum_{i=1}^{n} \log \frac{\mathcal{Z}^{-}(\mathbf{y})}{\mathcal{Z}^{+}(\mathbf{y})} \mathbf{e}_{i},
$$

where $\mathcal{Z}^{+}(\mathbf{y})$ and $\mathcal{Z}^{-}(\mathbf{y})$ are given in Eq.(20). In order to better understand the relationship between Eqs.(21) and (22), we suggest to elaborate Eq.(20), as follows. First of all, we change the variables of integration according to $z_{i}=\exp \left(i k_{i}\right)$. So doing, when $-\pi \leq k_{i} \leq+\pi$, the complex variable $z_{i}$ assumes the values on the unit circle $\left|z_{i}\right|=1$. This change of variable yields $k_{i}=-i \log z_{i}, \cos k_{i}=\frac{1}{2}\left(z_{i}+\frac{1}{z_{i}}\right)$ and $d k_{i}=\frac{1}{i} \frac{d z_{i}}{z_{i}}$. Therefore, Eq.(20) assumes the new form

$$
\mathcal{Z}^{ \pm}(\mathbf{y})=\frac{1}{i^{n}} \oint_{\{|z|=1\}^{n} \subset \mathbb{C}^{n}} \exp \left\{m\left[\sum_{i=1}^{n} y_{i} \log z_{i}+\log \sum_{i=1}^{n} \frac{1}{2}\left(z_{i}+\frac{1}{z_{i}}\right)\right]\right\} z_{i}^{ \pm 1} \prod_{k=1}^{n} z_{k}^{-1} d \mathbf{z},
$$

where the integral is performed over the collection of $n$ unit circles in $\mathbb{C}^{n}$ ( $n$-fold product of contours in the complex planes). Since the only singular points of the function within the integral are $z_{i}=0$ $\forall i=1, \ldots, n$, the contours can be freely modified on the complex planes, provided that they are always closed paths containing the origin of the axes. This possibility will be exploited to adopt an asymptotic technique to evaluate the integrals for large values of $m$. In particular, we will adopt the multivariate saddle point method [49-52]. The integral to study is of the form

$$
\mathcal{I}_{m}=\int_{M \subset \mathbb{C}^{n}} f(\mathbf{z}) \exp [m S(\mathbf{z})] d \mathbf{z},
$$


where $\mathbf{z}=\left(z_{1}, \ldots, z_{n}\right) \in \mathbb{C}^{n}$ and $f(\mathbf{z})$ and $S(\mathbf{z})$ are holomorphic functions over $\Gamma \subset \mathbb{C}^{n}$ (with $M \subset \Gamma$ ). We give here the main result of the multivariate saddle point method. We suppose that a point $\mathbf{z}_{0}=\left(z_{01}, \ldots, z_{0 n}\right) \in M$ exists such that:

1. $\mathbf{z}_{0}$ is a non-degenerate critical point (or saddle point) for $S(\mathbf{z})$, which is to say that $S^{\prime}\left(\mathbf{z}_{0}\right)=0$ and $\operatorname{det}\left[S^{\prime \prime}\left(\mathbf{z}_{0}\right)\right] \neq 0$, where $S^{\prime}(\mathbf{z})=\left(\frac{\partial S(\mathbf{z})}{\partial z_{1}}, \ldots, \frac{\partial S(\mathbf{z})}{\partial z_{n}}\right)$ and $S^{\prime \prime}(\mathbf{z})$ is the complex Hessian matrix containing the mixed derivatives, i.e. $S_{i j}^{\prime \prime}(\mathbf{z})=\frac{\partial^{2} S(\mathbf{z})}{\partial z_{i} \partial z_{j}}$;

2. the quantity $\max _{\mathbf{z} \in M} \Re \mathrm{e}\{S(\mathbf{z})\}$ is attained at $\mathbf{z}_{0} \in M$ (with $\mathbf{z}_{0}$ interior point of $M$ ).

If these conditions are satisfied, we have the asymptotic representation of the integral in Eq.(24), as follows

$$
\mathcal{I}_{m} \simeq\left(\frac{2 \pi}{m}\right)^{n / 2} \exp \left[m S\left(\mathbf{z}_{0}\right)\right] f\left(\mathbf{z}_{0}\right)\left\{\operatorname{det}\left[-S^{\prime \prime}\left(\mathbf{z}_{0}\right)\right]\right\}^{-1 / 2}
$$

where, if necessary, the last term can be directly evaluated by means of the eigenvalues $\mu_{j}(\forall j=1, \ldots, n)$ of the matrix $S^{\prime \prime}\left(\mathbf{z}_{0}\right)$

$$
\left\{\operatorname{det}\left[-S^{\prime \prime}\left(\mathbf{z}_{0}\right)\right]\right\}^{-1 / 2}=\exp \left[-\frac{i}{2} \sum_{j=1}^{n} \arg \left(-\mu_{j}\right)\right] \prod_{j=1}^{n}\left|\mu_{j}\right|^{-1 / 2} .
$$

For the application of the theorem, we have firstly to solve the equation $S^{\prime}\left(\mathbf{z}_{0}\right)=0$ in order to find the critical or saddle point of the function $S$. Then, we have to modify the contours on the complex planes in such a way that they pass through $\mathbf{z}_{\mathbf{0}}$. To do this, we have also to fix the direction of the path at the critical point. We adopt the following procedure. Since $S(\mathbf{z})$ is an holomorphic function, the real and imaginary parts $\Re \mathrm{e}\{S(\mathbf{z})\}$ and $\Im \mathrm{m}\{S(\mathbf{z})\}$ are harmonic functions and, therefore, the critical points are necessarily saddle points (or minimax points) for $\Re \mathrm{e}\{S(\mathbf{z})\}$ and $\Im \mathrm{m}\{S(\mathbf{z})\}$. It means that on each complex plane there is a direction, passing through the critical point, where $\Re$ e $\{S(\mathbf{z})\}$ has a maximum point and, at the same time $\Im \mathrm{m}\{S(\mathbf{z})\}$ is constant. This is exactly the direction that we must choose for the modified paths, passing through the critical point. Once deformed the $n$-fold product of contours in the complex planes as described above, the conditions of the multivariate saddle point method are satisfied and Eq.(25) can be used. In our case we can identify

$$
S(\mathbf{z})=\sum_{i=1}^{n} y_{i} \log z_{i}+\log \sum_{i=1}^{n} \frac{1}{2}\left(z_{i}+\frac{1}{z_{i}}\right),
$$

and we can define

$$
f^{ \pm}(\mathbf{z})=z_{i}^{ \pm 1} \prod_{k=1}^{n} z_{k}^{-1}
$$

so that Eq.(23) assumes the form

$$
\mathcal{Z}^{ \pm}(\mathbf{y})=\frac{1}{i^{n}} \oint_{\{|z|=1\}^{n} \subset \mathbb{C}^{n}} f^{ \pm}(\mathbf{z}) \exp [m S(\mathbf{z})] d \mathbf{z}
$$

similar to Eq.(24). We can identify the critical points as $\frac{\partial S}{\partial z_{k}}=0$, leading to the expression

$$
y_{k}=-\frac{z_{0 k}-\frac{1}{z_{0 k}}}{\sum_{i=1}^{n}\left(z_{0 i}+\frac{1}{z_{0 i}}\right)} \forall k=1, \ldots, n .
$$

This result defines only implicitly the critical point $\mathbf{z}_{0}$, since it can not be solved in closed form for obtaining its actual value. Anyway, we can follow the above procedure to deform the paths on the 
complex planes in order to fulfil the conditions of the multivariate saddle point method. The asymptotic representation can be eventually obtained in the form

$$
\mathcal{Z}^{ \pm}(\mathbf{y}) \simeq\left(\frac{2 \pi}{m}\right)^{n / 2} \exp \left[m S\left(\mathbf{z}_{0}\right)\right] f^{ \pm}\left(\mathbf{z}_{0}\right)\left\{\operatorname{det}\left[-S^{\prime \prime}\left(\mathbf{z}_{0}\right)\right]\right\}^{-1 / 2}
$$

Since we are interested in the thermodynamic limit of the Helmholtz force-extension relation, we substitute Eq.(31) in Eq.(22), thus obtaining

$$
\langle\boldsymbol{\eta}\rangle=\frac{1}{2} \sum_{i=1}^{n} \log \frac{\mathcal{Z}^{-}(\mathbf{y})}{\mathcal{Z}^{+}(\mathbf{y})} \mathbf{e}_{i} \simeq \frac{1}{2} \sum_{i=1}^{n} \log \frac{f^{-}\left(\mathbf{z}_{0}(\mathbf{y})\right)}{f^{+}\left(\mathbf{z}_{0}(\mathbf{y})\right)} \mathbf{e}_{i}
$$

where $\mathbf{z}_{0}(\mathbf{y})$ is implicitly defined through Eq.(30). Now, we find from Eq.(28) that $f^{-}\left(\mathbf{z}_{0}(\mathbf{y})\right) / f^{+}\left(\mathbf{z}_{0}(\mathbf{y})\right)=$ $z_{0 i}(\mathbf{y})^{-2}$ and, therefore, the force-extension relation for large values of $m$ is given by

$$
\langle\boldsymbol{\eta}\rangle \simeq \frac{1}{2} \sum_{i=1}^{n} \log \frac{1}{z_{0 i}(\mathbf{y})^{2}} \mathbf{e}_{i}=-\sum_{i=1}^{n} \log \left[z_{0 i}(\mathbf{y})\right] \mathbf{e}_{i} .
$$

It means that within the Helmholtz ensemble, for large values of $m$, each component of the normalized force can be written in term of the normalised extension as $\left\langle\eta_{k}\right\rangle=-\log \left[z_{0 k}(\mathbf{y})\right]$ where $z_{0 k}(\mathbf{y})$ is defined through Eq.(30). Since Eq.(30) gives the explicit form of $\mathbf{y}$ as function of $\mathbf{z}_{0}$ is more advisable to consider the inverse force extension relation given by $z_{0 k}(\mathbf{y})=\exp \left(-\left\langle\eta_{k}\right\rangle\right)$. Now, the latter expression can be substituted in Eq.(30) to give

$$
\lim _{m \rightarrow \infty} \mathbf{y}=\frac{\sum_{i=1}^{n} \sinh \left\langle\eta_{i}\right\rangle \mathbf{e}_{i}}{\sum_{i=1}^{n} \cosh \left\langle\eta_{i}\right\rangle},
$$

which exactly corresponds to Eq.(21), describing the response within the isotensional ensemble. Hence, we proved the ensemble equivalence in the thermodynamic limit. The correspondence of the forceextension responses under Gibbs and Helmholtz ensembles also justify a posteriori the introduction of the finite difference in place of the partial derivative in Eq.(17). The ensembles equivalence, here obtained for polymer lattice model, is in perfect agreement with the properties of continuous polymer models, as described in recent literature [20,21,26,27,29].

We presented here a general proof of the ensembles equivalence in the thermodynamic limit, which is valid for any dimension of the space embedding the stochastic process. However, for the particular cases of one-dimensional and two-dimensional spaces, the equivalence can be proved by means of a very simpler method, as described in Appendices A and B, respectively. Indeed, in these specific cases the Helmholtz partition function can be written in closed form through simple expressions, see Eqs.(61) and (73), which allows for the natural extension to real arguments, thus enabling the use of Eq.(16) for determining the force-extension curve (see Appendices A and B for details). For three-dimensional (or higher-dimensional) systems this simplified procedure cannot be applied, and we necessarily have to follow the general method introduced in the present section. This point is further explained in Appendix $\mathrm{C}$, where the three-dimensional case is thoroughly analysed. In particular, a closed form expression for the Helmholtz partition function is here obtained for $n=3$, see Eq.(87), and the impossibility to extend it to real arguments is discussed in detail. In Fig.2 we compare the force-extension responses for the uni-axial stretching of one-dimensional, two dimensional and three-dimensional systems (as obtained in Appendices A, B and C). For each dimensionality we show both the Gibbs and the Helmholtz response (for $m=80$ ). The difference between isotensional and isometric behaviors can be appreciated when we are far from the thermodynamic limit. The knowledge of the analytical solution for the case with $n=3$, as discussed in Appendix C, allows us to draw a comparison between the Gibbs response given in Eq.(80) and the Helmholtz one stated in Eqs.(88) and (89), as shown in Fig.3. Here, we observe the convergence of the force-extension curves for large values of $m$, coherently with the ensembles equivalence in the thermodynamic limit. 


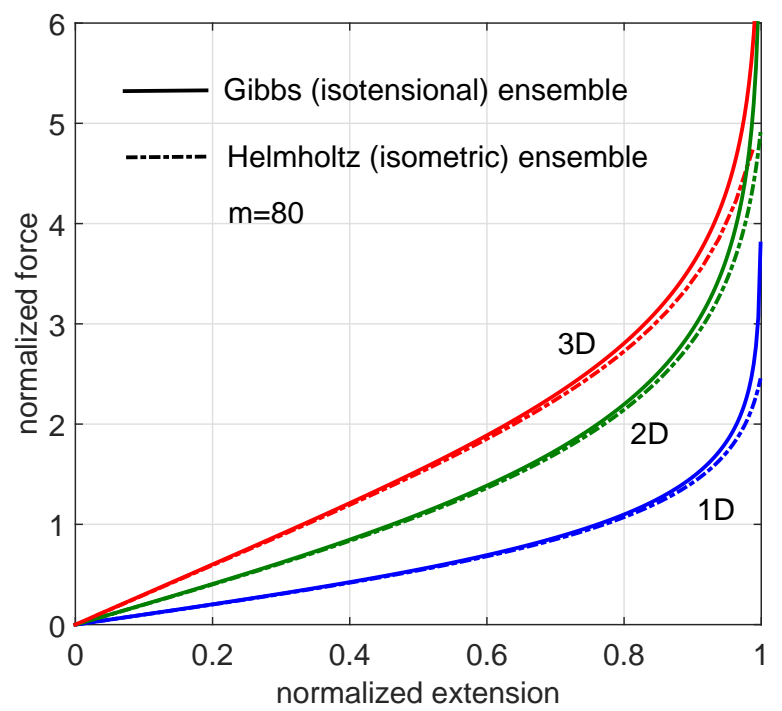

Fig. 2 Normalized force $\eta$ versus normalized extension $y$ for the uni-axial stretching of one-dimensional, two-dimensional and three-dimensional systems under both isotensional and isometric conditions (Gibbs and Helmholtz ensembles, respectively). While the Gibbs curves are independent of the number $m$ of elements of the chain, for the Helmholtz curves we adopted $m=80$.

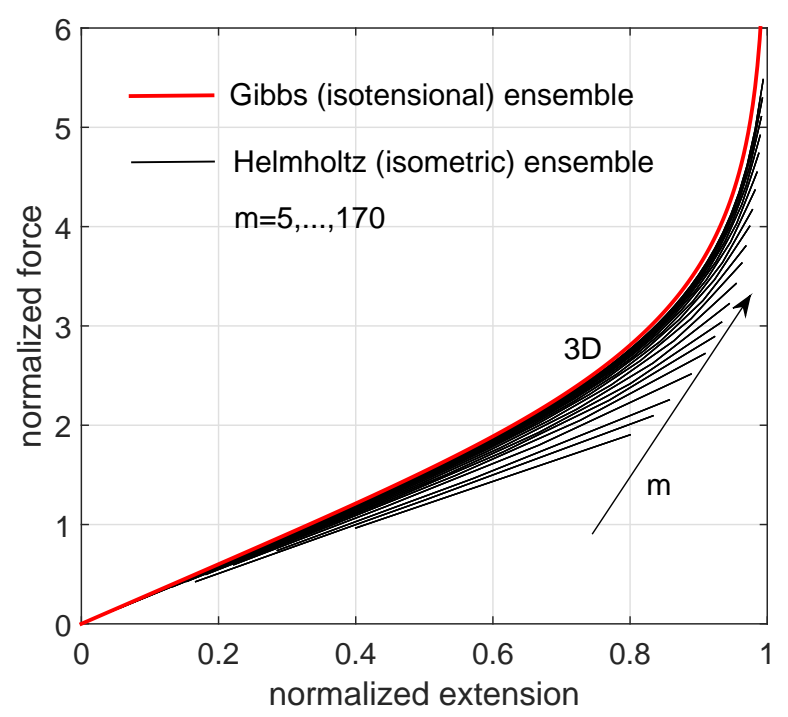

Fig. 3 Normalized force $\eta$ versus normalized extension $y$ for the uni-axial stretching applied to a threedimensional system under Gibbs isotensional conditions and Helmholtz isometric ones with several chain lengths. We used 20 integer values $m=5,6,7,9,11,13,15,18,22,27,32,39,46,56,67,81,97,117,141,170$, distributed in geometric progression. The convergence of the curves towards the Gibbs response represents the ensembles equivalence in the thermodynamic limit.

\section{Bistability in polymer lattice models}

In this section we generalize the concepts of Gibbs ensemble, Helmholtz ensemble and thermodynamic limit for polymers that may undergo conformational transitions described by a bistable behavior. In other word, the segments defining the polymer structure may assume two different lengths, namely $\ell$ and $\chi \ell$, where $\chi$ is an integer (being the polymer always confined on a lattice). The two configurations of each units correspond to different energetic contribution entering the total Hamiltonian of the system. 
In particular, the folded state (length $\ell$ ) is associate with the energy $g_{f}$ and the unfolded one (length $\chi \ell$ ) with the energy $g_{u}>g_{f}$. Since the unfolding is energetically unfavourable, the transition from unfolded to folded state can be promoted by an applied force (isotensional conditions) or a prescribed stretching (isometric conditions). This approach is useful to model the folding and unfolding of polymer chains, including those of biological origin. This point is particularly important since the recent introduction of several single-molecule force spectroscopy techniques has allowed the direct measuring of the elastic response of individual molecules, thus investigating the statistical mechanics of small systems.

We remark that in our model we assumed to have a single rigid segment after the unfolding process. Of course, this is an approximation, which do not correspond to the reality of several macromolecules. A more refined model should consider unfolded elements acting as a flexible sub-chain composed of $\chi$ segments of length $\ell$. However, the unfolding events occur when the macromolecular chain is sufficiently stretched and, therefore, the system is quite aligned to the direction of the applied stretching. So, in this condition the approximation introduced is not too severe. Anyway, from the statistical mechanics point of view, the generalization with flexible unfolded units is interesting and will be considered in a future investigation.

\subsection{The Gibbs ensemble for bistable systems}

We consider a polymer with $m$ bistable segments randomly arranged on the $\mathbb{Z}^{n}$ lattice, each of length $\ell$ or $\chi \ell$. As before, the positions of the elements are $\mathbf{x}_{1}, \ldots, \mathbf{x}_{m}$ and the Hamiltonian of the system is now given by

$$
\mathcal{H}=n_{f} g_{f}+n_{u} g_{u}-\mathbf{f} \cdot \mathbf{x}_{m} .
$$

where $n_{f}$ is the number of folded units, $n_{u}$ is the number of unfolded ones and $\mathbf{f}$ is the applied force. Each segment of the lattice polymer is represented by a vector $\ell \boldsymbol{\sigma}_{i} \forall i=1, \ldots, m$, where the vectors $\boldsymbol{\sigma}_{i}$ assume their values from the set $\Theta=\left\{ \pm \mathbf{e}_{k}, \pm \chi \mathbf{e}_{k}, k=1, \ldots, n\right\}=\Theta_{f} \cup \Theta_{u}$. Here, $\Theta_{f}=\left\{ \pm \mathbf{e}_{k}, k=1, \ldots, n\right\}$ and $\Theta_{u}=\left\{ \pm \chi \mathbf{e}_{k}, k=1, \ldots, n\right\}$. Moreover, $\Sigma$ is the sequence $\left(\boldsymbol{\sigma}_{1}, \ldots, \boldsymbol{\sigma}_{m}\right) \in \Theta^{m}$. Of course, we have $\mathbf{x}_{m}=\ell \sum_{i=1}^{m} \boldsymbol{\sigma}_{i}$. The canonical distribution within the Gibbs ensemble is then given by

$$
P_{G}(\Sigma)=\frac{1}{Z_{G}(\mathbf{f})} \exp \left(-\frac{n_{f} g_{f}+n_{u} g_{u}}{k_{B} T}\right) \exp \left(\frac{\ell}{k_{B} T} \mathbf{f} \cdot \sum_{i=1}^{m} \boldsymbol{\sigma}_{i}\right)
$$

where the partition function $Z_{G}$ can be defined as follows

$$
\begin{aligned}
Z_{G}(\mathbf{f}) & =\sum_{\Sigma \in \Omega} \exp \left(-\frac{n_{f} g_{f}+n_{u} g_{u}}{k_{B} T}\right) \exp \left(\frac{\ell}{k_{B} T} \mathbf{f} \cdot \sum_{i=1}^{m} \boldsymbol{\sigma}_{i}\right) \\
& =2^{m}\left[\exp \left(-\frac{g_{f}}{k_{B} T}\right) \sum_{i=1}^{n} \cosh \left(\frac{\ell f_{i}}{k_{B} T}\right)+\exp \left(-\frac{g_{u}}{k_{B} T}\right) \sum_{i=1}^{n} \cosh \left(\frac{\chi \ell f_{i}}{k_{B} T}\right)\right]^{m} .
\end{aligned}
$$

The constitutive equation of the system can be obtained through Eq.(13) and we eventually get

$$
\left\langle\mathbf{x}_{m}\right\rangle=m \ell \frac{\sum_{i=1}^{n}\left[\mathrm{e}^{\frac{\Delta}{k_{B} T}} \sinh \left(\frac{\ell f_{i}}{k_{B} T}\right)+\chi \sinh \left(\frac{\chi \ell f_{i}}{k_{B} T}\right)\right] \mathbf{e}_{i}}{\sum_{i=1}^{n}\left[\mathrm{e}^{\frac{\Delta}{k_{B} T}} \cosh \left(\frac{\ell f_{i}}{k_{B} T}\right)+\cosh \left(\frac{\chi \ell f_{i}}{k_{B} T}\right)\right]},
$$

where $\Delta=g_{u}-g_{f}$ is the energy jump between the folded and unfolded configurations.

It is interesting to note that a similar expression has been recently obtained for a different onedimensional model composed of a chain of continuous elements (not disposed on a lattice) with mechanical bistability, i.e. with a multi-basin energy landscape [34]. The model was solved by the introduction of a set of spin variables, able to identify the basin explored by each element of the chain. For large effective mechanical stiffness of the wells, the behavior of this model is identical to the lattice system here introduced. It is also important to remark that this behavior, characterized by a plateau force in the force-extension response, is typical of several polymer structures of biological origin. For example, the plateau-like response has been observed for double-stranded DNA [53,54] and it has been 

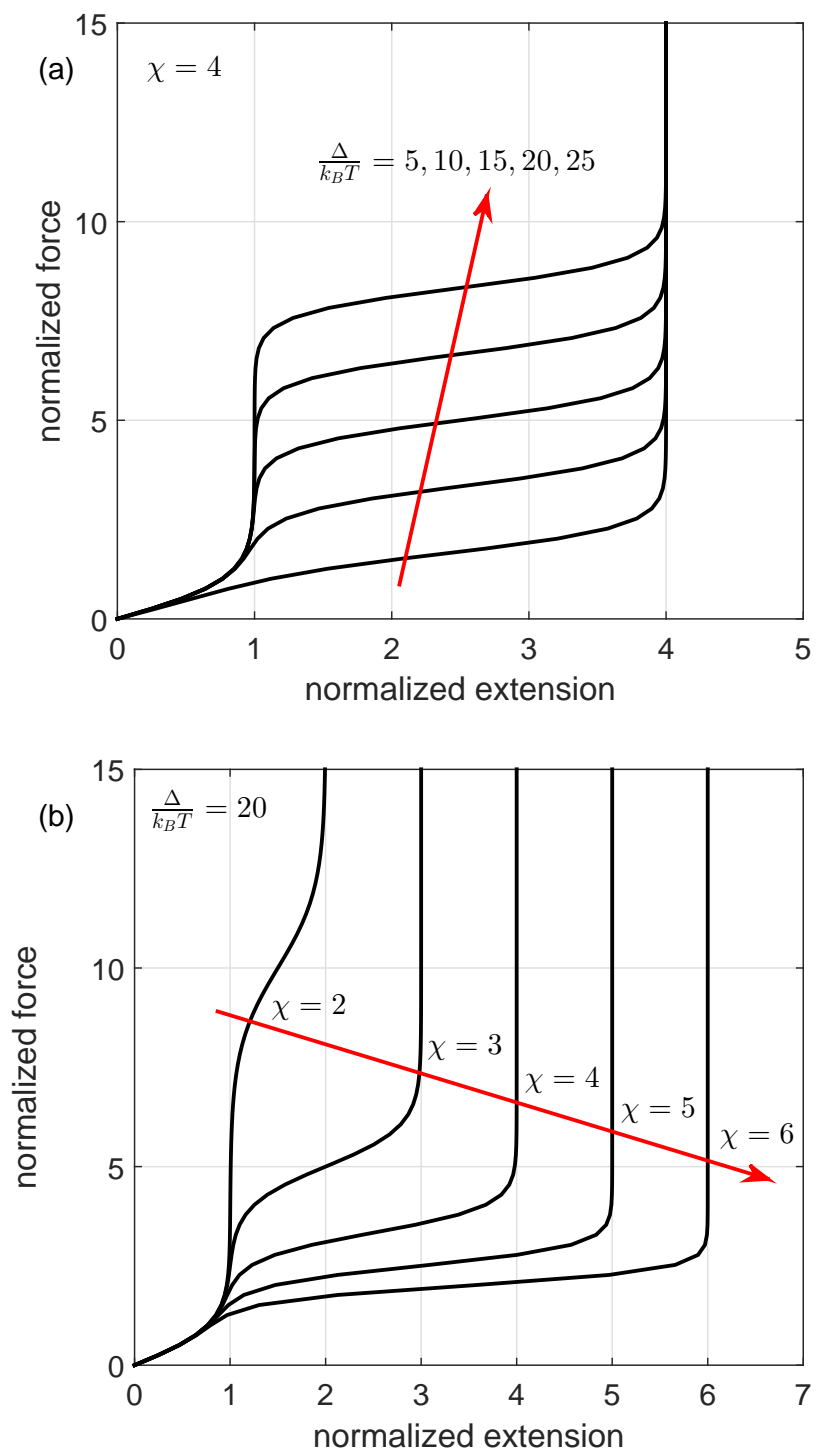

Fig. 4 Normalized force $\eta$ versus normalized extension $y$ for the stretching of a one-dimensional system $(n=1)$ with conformational transitions and within the Gibbs ensemble. While in panel (a) we fixed the elongation factor $\chi=4$ and we varied the normalized energy jump $\frac{\Delta}{k_{B} T}=5,10,15,20,25$, in panel (b) we fixed $\frac{\Delta}{k_{B} T}=20$ and we varied $\chi=2,3,4,5,6$. In any case, we can observe a plateau force at $\eta^{*}=\frac{f^{*} \ell}{k_{B} T}=\frac{\Delta}{(\chi-1) k_{B} T}$ characterizing the cooperative or simultaneous unfolding of the elements.

studied through thermodynamic approaches $[55,56]$ and by other statistical analyses, leading to the melting interpretation of the transition [57] or to the explanation of the transition through a new structure named stretched-DNA, or S-DNA [58]. The plateau-like response has been also observed for long polysaccharides, such as dextran [28,59], and conveniently interpreted by means of a continuous two-state model [60].

Some examples of force-extension responses generated by Eq.(38) are represented in Fig.4. Here, we considered the behavior of the system with $n=1$ under stretching and we plotted the normalized force $\eta=\frac{f \ell}{k_{B} T}$ versus the normalized extension $y=\frac{\left\langle x_{m}\right\rangle}{m \ell}$. While in Fig.4(a) we fixed the elongation factor $\chi$ and we varied the normalized energy jump $\frac{\Delta}{k_{B} T}$, in Fig.4(b) we fixed $\frac{\Delta}{k_{B} T}$ and we varied $\chi$. In all cases, we observe a force plateau corresponding to $f^{*}=\frac{\Delta}{(\chi-1) \ell}$ or, equivalently, $\frac{f^{*} \ell}{k_{B} T}=\frac{\Delta}{(\chi-1) k_{B} T}$ 




Fig. 5 Effect of the system dimensionality on the Gibbs response of systems with conformational transitions. The normalized force $\eta$ is shown versus the normalized extension $y$ for describing the stretching of systems with $n=1,2,3$. We fixed the elongation factor $\chi=3$ and the normalized energy jump $\frac{\Delta}{k_{B} T}=20$. In any case, we can observe that the plateau force at $\eta^{*}=\frac{f^{*} \ell}{k_{B} T}=\frac{\Delta}{(\chi-1) k_{B} T}$ is independent of the system dimensionality.

(normalized quantity). The interpretation of this behavior is based on a cooperative process inducing the simultaneous transition of all elements of the chain at the same value of force $f^{*}$. The value of the threshold force $f^{*}$ can be explained as follows. If we consider one element of the chain under the application of the force $f$, we have the two possible potential energies $U_{f}=g_{f}-f \ell$ and $U_{u}=g_{u}-f \chi \ell$, corresponding to the folded and unfolded states, respectively. Hence, the unfolded configuration is more stable of the folded one if and only if $U_{u}<U_{f}$ that is to say $f<\frac{\Delta}{(\chi-1) \ell}$, as observed in Fig.4. Notably, the value of the plateau force inducing the conformation transition does not depend on the temperature. Such a result is readily interpreted in the framework of the Bell expression, originally derived in the context of the adhesion of cells [61]. Moreover, the threshold force characterising the cooperative transition is in perfect agreement with some other continuous models, recently introduced in literature [29,34].

To conclude, we show in Fig.5 the effect of the dimensionality on the force-extension response under the Gibbs conditions. We can affirm that the plateau force observed in the $\eta-y$ curve, and above discussed, is independent of the dimensionality of the system, being only the slope of the first part of the curve affected by $n$. This is coherent with Fig.2, showing the force-extension curve for systems without conformational transitions.

\subsection{The Helmholtz ensemble for bistable systems}

In order to correctly define the Helmholtz partition function for systems with conformational transitions, we have to better analyse the random paths on the $\mathbb{Z}^{n}$ lattice characterized by two type of steps (bistability). Hence, we consider one standard step with extension 1 and one elongated step with extension $\chi \in \mathbb{N}$. The probability of having a standard step is $p_{f}$ and the probability of having an elongated step is $p_{u}$. Of course, these probabilities are proportional to the Boltzmann weights calculated with the folding and unfolding energies $g_{f}$ and $g_{u}$, respectively. It means that $p_{f}=\alpha \exp \left(-\frac{g_{f}}{k_{B} T}\right)$ and $p_{u}=\alpha \exp \left(-\frac{g_{u}}{k_{B} T}\right)$. Since we have to respect the obvious relation $2 n p_{f}+2 n p_{u}=1$, we can obtain the value of $\alpha$ and the explicit form for the folding and unfolding probabilities is given by

$$
p_{f}=\frac{1}{2 n} \frac{\exp \left(\frac{\Delta}{k_{B} T}\right)}{1+\exp \left(\frac{\Delta}{k_{B} T}\right)} \text { and } p_{u}=\frac{1}{2 n} \frac{1}{1+\exp \left(\frac{\Delta}{k_{B} T}\right)},
$$


where, as before, $\Delta=g_{u}-g_{f}$. We remark that if $\Delta \rightarrow \infty$ we obtain $p_{f} \rightarrow 1 /(2 n)$ and $p_{u} \rightarrow 0$, which corresponds to the case without conformational transitions (see Section 2). The equation governing the dynamics of the probabilities $P_{m}(\mathbf{x})$ for the random path on the $\mathbb{Z}^{n}$ lattice can be now written in the following form

$$
P_{m+1}(\mathbf{x})=p_{f} \sum_{i=1}^{n}\left[P_{m}\left(\mathbf{x}-\mathbf{e}_{i}\right)+P_{m}\left(\mathbf{x}+\mathbf{e}_{i}\right)\right]+p_{u} \sum_{i=1}^{n}\left[P_{m}\left(\mathbf{x}-\chi \mathbf{e}_{i}\right)+P_{m}\left(\mathbf{x}+\chi \mathbf{e}_{i}\right)\right],
$$

which represents a simple generalization of Eq.(1). We can perform the same calculations developed in Section 2. We define the generating function exactly as in Eq.(2), and we find its evolution equation

$$
z \mathcal{P}(\mathbf{x}, z)-z P_{0}(\mathbf{x})=p_{f} \sum_{i=1}^{n}\left[\mathcal{P}\left(\mathbf{x}-\mathbf{e}_{i}, z\right)+\mathcal{P}\left(\mathbf{x}+\mathbf{e}_{i}, z\right)\right]+p_{u} \sum_{i=1}^{n}\left[\mathcal{P}\left(\mathbf{x}-\chi \mathbf{e}_{i}, z\right)+\mathcal{P}\left(\mathbf{x}+\chi \mathbf{e}_{i}, z\right)\right]
$$

which is a generalization of Eq.(3). By adopting the same method described in Section 2, we eventually obtain the solution

$$
\mathcal{P}(\mathbf{x}, z)=\frac{1}{(2 \pi)^{n}} \int_{-\pi}^{+\pi} \ldots \int_{-\pi}^{+\pi} \frac{\mathrm{e}^{i \mathbf{k} \cdot \mathbf{x}} d \mathbf{k}}{1-2 p_{f} z^{-1} \sum_{i=1}^{n} \cos k_{i}-2 p_{u} z^{-1} \sum_{i=1}^{n} \cos \chi k_{i}},
$$

corresponding to the initial condition $P_{0}(\mathbf{x})=\delta(\mathbf{x})$. This expression represents a generalized form of the lattice Green function. From this solution, by means of the property stated in Eq.(6), we get the occupation probability

$$
P_{m}(\mathbf{x})=\frac{2^{m-n}}{\pi^{n}} \int_{-\pi}^{+\pi} \cdots \int_{-\pi}^{+\pi} \mathrm{e}^{i \mathbf{k} \cdot \mathbf{x}}\left[p_{f} \sum_{i=1}^{n} \cos k_{i}+p_{u} \sum_{i=1}^{n} \cos \chi k_{i}\right]^{m} d \mathbf{k} .
$$

This result represent the probability of arriving in $\mathbf{x}$ at step $m$ for a random path with bistable segments.

As already introduced in Section 6.1, each segment of the lattice polymer is represented by a vector $\ell \boldsymbol{\sigma}_{i} \forall i=1, \ldots, m$, where the vectors $\boldsymbol{\sigma}_{i}$ assume their values from the set $\Theta=\left\{ \pm \mathbf{e}_{k}, \pm \chi \mathbf{e}_{k}, k=1, \ldots, n\right\}=$ $\Theta_{f} \cup \Theta_{u}$. Here, $\Theta_{f}=\left\{ \pm \mathbf{e}_{k}, k=1, \ldots, n\right\}$ and $\Theta_{u}=\left\{ \pm \chi \mathbf{e}_{k}, k=1, \ldots, n\right\}$. Besides, we define $\Omega$ as the space of all the possible sequences of segments, $\Omega=\left\{\left(\boldsymbol{\sigma}_{1}, \ldots, \boldsymbol{\sigma}_{m}\right) \in \Theta^{m}\right\}$. In the Helmholtz or isometric ensemble, both the end-terminals of the polymer chain are tethered, the first at $\mathbf{0}$ and second at $\mathbf{x} \in \mathbb{Z}^{n}$ (corresponding to the physical value $\mathbf{x}_{m}=\ell \mathbf{x}$ ). Hence, we define $\Omega_{0}$ as the space of all the sequences respecting the Helmholtz constraints, $\Omega_{0}=\left\{\left(\boldsymbol{\sigma}_{1}, \ldots, \boldsymbol{\sigma}_{m}\right) \in \Theta^{m}: \sum_{j=1}^{m} \boldsymbol{\sigma}_{j}=\mathbf{x}\right\}$, i.e. arriving in $\mathbf{x}$ at step $m$. Within the Helmholtz ensemble, the Hamiltonian of the polymer chain is simply given by the sum of the folding and unfolding energies, namely

$$
\mathcal{H}=n_{f} g_{f}+n_{u} g_{u} .
$$

If we consider the space $\Omega$, the probability of obtaining a given sequence $\Sigma=\left(\boldsymbol{\sigma}_{1}, \ldots, \boldsymbol{\sigma}_{m}\right)$ is defined as

$$
P_{F}(\Sigma)=\frac{\exp \left(-\frac{\mathcal{H}}{k_{B} T}\right)}{Z_{F}} \text { where } Z_{F}=\sum_{\Sigma \in \Omega} \exp \left(-\frac{\mathcal{H}}{k_{B} T}\right)
$$

Here, the subscript $F$ stands for free since we have no constraints on the chain configuration.

On the other hand, if we consider the space $\Omega_{0}$, the probability of obtaining a given sequence $\Sigma=\left(\boldsymbol{\sigma}_{1}, \ldots, \boldsymbol{\sigma}_{m}\right)\left(\right.$ with $\left.\sum_{j=1}^{m} \boldsymbol{\sigma}_{j}=\mathbf{x}\right)$ is defined as

$$
P_{H}(\Sigma)=\frac{\exp \left(-\frac{\mathcal{H}}{k_{B} T}\right)}{Z_{H}(\mathbf{x})} \text { where } Z_{H}(\mathbf{x})=\sum_{\Sigma \in \Omega_{0}} \exp \left(-\frac{\mathcal{H}}{k_{B} T}\right)
$$


Here, the subscript $H$ stands for Helmholtz and we have the above discussed constraint on the chain configuration (prescribed extension). We observe that the domains of definition of $P_{F}$ and $P_{H}$ are different (being $\Omega$ and $\Omega_{0}$, respectively) and, therefore, the numerical values of $Z_{F}$ and $Z_{H}$ are different.

Now, we have to investigate the relation between $P_{m}(\mathbf{x})$ and the Helmholtz partition function $Z_{H}(\mathbf{x})$. The probability to be in $\mathbf{x}$ at step $m$ is exactly the sum of all probabilities pertaining to all chains starting at $\mathbf{0}$ and arriving at $\mathbf{x}$. It means that

$$
P_{m}(\mathbf{x})=\sum_{\Sigma \in \Omega_{0}} P_{F}(\Sigma)
$$

Now, it is not difficult to draw an important conclusion

$$
P_{m}(\mathbf{x})=\frac{1}{Z_{F}} \sum_{\Sigma \in \Omega_{0}} \exp \left(-\frac{\mathcal{H}}{k_{B} T}\right)=\frac{Z_{H}(\mathbf{x})}{Z_{F}}
$$

where we used Eqs.(45) and (46). Hence, we obtain $Z_{H}(\mathbf{x})=Z_{F} P_{m}(\mathbf{x})$ and since $Z_{F}=Z_{G}(\mathbf{0})$, see Eq.(37), we finally get the expression

$$
Z_{H}(\mathbf{x})=Z_{G}(\mathbf{0}) P_{m}(\mathbf{x})=(2 n)^{m}\left[\exp \left(-\frac{g_{f}}{k_{B} T}\right)+\exp \left(-\frac{g_{u}}{k_{B} T}\right)\right]^{m} P_{m}(\mathbf{x})
$$

To conclude, this expression represents the partition function for a bistable polymer chain within the Helmholtz or isometric ensemble, where $P_{m}(\mathbf{x})$ is given in Eq.(43). We remark that if $g_{u} \rightarrow \infty$ and $g_{f} \rightarrow 0$ we lose the bistability and Eq.(49) reduces to Eq.(8), as expected. Of course, the force-extension relation for bistable systems within the Helmholtz ensemble is described again by Eq.(17).

We specialize now this result to the case of one-dimensional systems, i.e. to $n=1$. Hence, Eq.(43) simplifies delivering

$$
\begin{aligned}
P_{m}(x) & =\frac{2^{m}}{2 \pi} \int_{-\pi}^{+\pi} \mathrm{e}^{i k x}\left[p_{f} \cos k+p_{u} \cos \chi k\right]^{m} d k \\
& =\frac{1}{2 \pi} \sum_{h=0}^{m} \sum_{p=0}^{h} \sum_{t=0}^{m-h}\left(\begin{array}{c}
m \\
h
\end{array}\right)\left(\begin{array}{c}
h \\
p
\end{array}\right)\left(\begin{array}{c}
m-h \\
t
\end{array}\right) p_{u}^{h} p_{f}^{m-h} \int_{-\pi}^{+\pi} \mathrm{e}^{i k(x-h \chi+2 p \chi-m+h+2 t)} d k \\
& =\sum_{h=0}^{m} \sum_{p=0}^{h} \sum_{t=0}^{m-h}\left(\begin{array}{c}
m \\
h
\end{array}\right)\left(\begin{array}{c}
h \\
p
\end{array}\right)\left(\begin{array}{c}
m-h \\
t
\end{array}\right) p_{u}^{h} p_{f}^{m-h} \delta(x-h \chi+2 p \chi-m+h+2 t),
\end{aligned}
$$

where $\delta(j)$ represents the discrete delta function, i.e. $\delta(0)=1$ and $\delta(j)=0$ if $j \neq 0$. The Helmholtz constitutive equation in terms of the normalized force $\langle\eta\rangle=\frac{\langle f\rangle \ell}{k_{B} T}$ and extension $y=\frac{x_{m}}{m \ell}$ finally reads as follows

$$
\langle\eta\rangle=\frac{1}{2} \log \frac{\sum_{h=0}^{m} \sum_{p=0}^{h} \sum_{t=0}^{m-h}\left(\begin{array}{c}
m \\
h
\end{array}\right)\left(\begin{array}{c}
h \\
p
\end{array}\right)\left(\begin{array}{c}
m-h \\
t
\end{array}\right) p_{u}^{h} p_{f}^{m-h} \delta(m y-1-h \chi+2 p \chi-m+h+2 t)}{\sum_{h=0}^{m} \sum_{p=0}^{h} \sum_{t=0}^{m-h}\left(\begin{array}{c}
m \\
h
\end{array}\right)\left(\begin{array}{c}
h \\
p
\end{array}\right)\left(\begin{array}{c}
m-h \\
t
\end{array}\right) p_{u}^{h} p_{f}^{m-h} \delta(m y+1-h \chi+2 p \chi-m+h+2 t)},
$$

and it may be compared with its Gibbs counterpart given in Eq.(38) (for $n=1$ ). This is done in Fig.6, where we may observe that the Helmholtz response is characterized by a non-cooperative or sequential unfolding of the chain elements. It means that the elements unfold progressively in response to the increasing extension. This peculiar behavior is perfectly consistent with a wide and consolidated knowledge, based on continuous models and single-molecule experiments $[28,59,62,63]$. It is therefore important to have established that this behavior can be also described by polymer lattice models.

In Fig.6 we observe that the last points of the curves correspond to a negative force. This is a specific behavior induced by the fact that we represented the response for the one-dimensional case and for small values of $m$. For $n=1$, the idea of a polymer as a random coil is degenerated to a simple distribution of segments on one axis with prescribed total length. The last points with negative force correspond to a situation where quite all the domains are unfolded and aligned and the few domains not yet unfolded try to unfold in response to the increasing extension. Since the lengths $\ell$ and $\chi \ell$ are fixed, is not difficult to imagine a pushing force (negative) applied to the device instead of 



Fig. 6 Comparison between Gibbs and Helmholtz responses for the stretching of a one-dimensional system $(n=1)$ with conformational transitions. The normalized force $\eta$ is represented versus the normalized extension $y$ for $\chi=4$ and $\frac{\Delta}{k_{B} T}=20$. We also used $m=10$ in panel (a) and $m=20$ in panel (b). While the Gibbs curve shows the cooperative or simultaneous unfolding of the elements, the Helmholtz one describes the noncooperative or sequential unfolding of the chain units.

a pulling one (positive). Of course, this apparently paradoxical behavior disappears for larger $m$ and, more importantly, for $n=2$ or 3 where the exploration of the phase space is more pertinent to polymer models.

In the literature, some continuous theories have been developed to explain the sawtooth-like response observed in several experiments. A statistical mechanics theory has been introduced through a suitable free energy, eventually yielding a series of first-order phase transitions in correspondence to the unfolding processes $[32,33]$. The minimization of the total energy of a two-state system is in good agreement with the sawtooth pattern observed in titin experiments [64]. Besides, the mechanical un- 
folding of proteins has been studied through the Ising model [65]. Finally, an exhaustive understanding of chain behaviors driven by hard or soft devices has been achieved by analysing discrete systems with folding/unfolding units [35,66-68], Fermi-Pasta-Ulam chains of bistable elements [69], and structures undergoing discrete phase transformations $[36,70,71]$.

A final comment concerns the plots shown in Fig.6. As one can see, the Helmholtz curves are limited to a given region of extension and they do not arrive at the maximum extension $x_{m}=m \chi \ell$ or, equivalently, $y=\chi$ (normalized quantity). This issue is related to the reachability problem concerning the paths on a lattice. Indeed, if we consider a path starting from the origin of the axes, composed of a fixed number of steps $m$, and able to make only specific moves, we have that only a subset of the lattice points are reachable and, therefore, the subset of unreachable points is non-empty. This is true for the polymers without conformational transition with moves within the set $\left\{ \pm \mathbf{e}_{k}, k=1, \ldots, n\right\}$, as evident in Fig.3, and also for polymers with conformational transitions with moves within $\left\{ \pm \mathbf{e}_{k}, \pm \chi \mathbf{e}_{k}, k=1, \ldots, n\right\}$, as shown in Fig.6. This is an intrinsic limitation of the Helmholtz conditions for polymers on a lattice, which however do not limits the applicability and the physical interpretation of the force-extension responses.

6.3 The ensemble equivalence for bistable systems

We are now interested in comparing the force-extension relations obtained under isotensional and isometric conditions for a large number of elements of the chain. To do this, it is better to introduce normalized quantities for forces and extensions. Since $\mathbf{x} \in \mathbb{Z}^{n}$ and $\mathbf{x}_{m}=\ell \mathbf{x} \in \Re^{n}$, we define the normalized length $\mathbf{y}=\mathbf{x}_{m} /(m \ell)$. On the other hand, we define the normalized force through the relation $\boldsymbol{\eta}=\frac{\ell \mathbf{f}}{k_{B} T}$. So doing, the force-extension relation within the Gibbs ensemble given in Eq.(38) assumes the simpler form

$$
\langle\mathbf{y}\rangle=\frac{\sum_{i=1}^{n}\left[\mathrm{e}^{\frac{\Delta}{k_{B} T}} \sinh \left(\eta_{i}\right)+\chi \sinh \left(\chi \eta_{i}\right)\right] \mathbf{e}_{i}}{\sum_{i=1}^{n}\left[\mathrm{e}^{\frac{\Delta}{k_{B} T}} \cosh \left(\eta_{i}\right)+\cosh \left(\chi \eta_{i}\right)\right]} .
$$

On the other hand, the constitutive equation within the Helmholtz ensembles given in Eq.(17) combined with Eq.(49) reads

$$
\langle\boldsymbol{\eta}\rangle=\frac{1}{2} \sum_{i=1}^{n} \log \frac{\mathcal{Z}^{-}(\mathbf{y})}{\mathcal{Z}^{+}(\mathbf{y})} \mathbf{e}_{i}
$$

where $\mathcal{Z}^{+}(\mathbf{y})$ and $\mathcal{Z}^{-}(\mathbf{y})$ are given by

$$
\mathcal{Z}^{ \pm}(\mathbf{y})=\int_{-\pi}^{+\pi} \cdots \int_{-\pi}^{+\pi} \exp \left\{m\left[i \mathbf{k} \cdot \mathbf{y}+\log \left(p_{f} \sum_{i=1}^{n} \cos k_{i}+p_{u} \sum_{i=1}^{n} \cos \chi k_{i}\right)\right]\right\} \exp \left( \pm i \mathbf{k} \cdot \mathbf{e}_{i}\right) d \mathbf{k} \cdot(54)
$$

Now, following the procedure introduced in Section 5, we change the variables of integration according to $z_{i}=\exp \left(i k_{i}\right)$. We eventually obtain

$$
\mathcal{Z}^{ \pm}(\mathbf{y})=\frac{1}{i^{n}} \oint_{\{|z|=1\}^{n} \subset \mathbb{C}^{n}} f^{ \pm}(\mathbf{z}) \exp [m S(\mathbf{z})] d \mathbf{z}
$$

where

$$
S(\mathbf{z})=\sum_{i=1}^{n} y_{i} \log z_{i}+\log \left[\sum_{i=1}^{n} \frac{p_{f}}{2}\left(z_{i}+\frac{1}{z_{i}}\right)+\sum_{i=1}^{n} \frac{p_{u}}{2}\left(z_{i}^{\chi}+\frac{1}{z_{i}^{\chi}}\right)\right]
$$

and

$$
f^{ \pm}(\mathbf{z})=z_{i}^{ \pm 1} \prod_{k=1}^{n} z_{k}^{-1}
$$






Fig. 7 Normalized force $\eta$ versus normalized extension $y$ for the stretching of a one-dimensional system $(n=1)$ with conformational transitions. We plotted both the Gibbs response and the Helmholtz one for $m=4, \ldots, 10$ with $\chi=4$ and $\frac{\Delta}{k_{B} T}=20$. The plot represents a zoom in the region of the first force peak to show the force reduction with increasing $m$, corresponding to the convergence to the thermodynamic limit.

As before, we can apply the multivariate saddle point method. We begin by determining the critical point $\mathbf{z}_{0}$ of $S(\mathbf{z})$. We elaborate the equation $\frac{\partial S}{\partial z_{k}}=0$, delivering

$$
y_{k}=-\frac{p_{f}\left(z_{0 k}-\frac{1}{z_{0 k}}\right)+p_{u} \chi\left(z_{0 k}^{\chi}-\frac{1}{z_{0 k}^{\chi}}\right)}{\sum_{i=1}^{n} p_{f}\left(z_{0 i}+\frac{1}{z_{0 i}}\right)+\sum_{i=1}^{n} p_{u}\left(z_{0 i}^{\chi}+\frac{1}{z_{0 i}^{\chi}}\right)} \forall k=1, \ldots, n,
$$

which gives $\mathbf{y}$ as function of $\mathbf{z}_{0}$. In order to apply the multivariate saddle point method, we have to modify the contours on the complex planes in such a way that they pass through $\mathbf{z}_{\mathbf{0}}$. The direction of the contours at $\mathbf{z}_{\mathbf{0}}$ is fixed by imposing that $\Re \mathrm{e}\{S(\mathbf{z})\}$ has a maximum point and, at the same time $\Im \mathrm{m}\{S(\mathbf{z})\}$ is constant. Hence, the conditions of validity of the saddle point method are satisfied and Eq. (25) can be used. So, by using Eq.(53) for large values of $m$, we have $\left\langle\eta_{k}\right\rangle=-\log \left[z_{0 k}(\mathbf{y})\right]$, where $z_{0 k}(\mathbf{y})$ is defined through Eq.(58). The latter expression is equivalent to $z_{0 k}(\mathbf{y})=\exp \left(-\left\langle\eta_{k}\right\rangle\right)$, which can be substituted in Eq.(58) to give the force-extension relation for $m \rightarrow \infty$

$$
y_{k}=\frac{\mathrm{e}^{\frac{\Delta}{k_{B} T}} \sinh \left(\left\langle\eta_{k}\right\rangle\right)+\chi \sinh \left(\chi\left\langle\eta_{k}\right\rangle\right)}{\sum_{i=1}^{n}\left[\mathrm{e}^{\frac{\Delta}{k_{B} T}} \cosh \left(\left\langle\eta_{i}\right\rangle\right)+\cosh \left(\chi\left\langle\eta_{i}\right\rangle\right)\right]} .
$$

This result is coincident with Eq.(52), thus proving the equivalence of the ensembles in the thermodynamic limit also in the case of bistable systems.

From the point of view of the force-extension curve, the convergence to the thermodynamic limit can be realized by observing that the height of the characteristic force peaks in the Helmholtz response is a decreasing function of the number $m$ of elements of the chain. So doing, the Helmholtz forceextension response can reach the continuous and monotone shape of the Gibbs response for large values of $m$. This can be seen in Fig.7, where one can find the force-extension behavior zoomed in the region of the first force peak of the Helmholtz curves (for $m=4, \ldots, 10$ ) and compared with the corresponding Gibbs curve. The shift of the peak position explains the force reduction, going towards the thermodynamic limit. This trend is further confirmed in Fig.8, where the height of the first six peaks of the force-extension response is represented versus the chain length $m$, numerically demonstrating the convergence to the thermodynamic limit, as previously proved theoretically. 


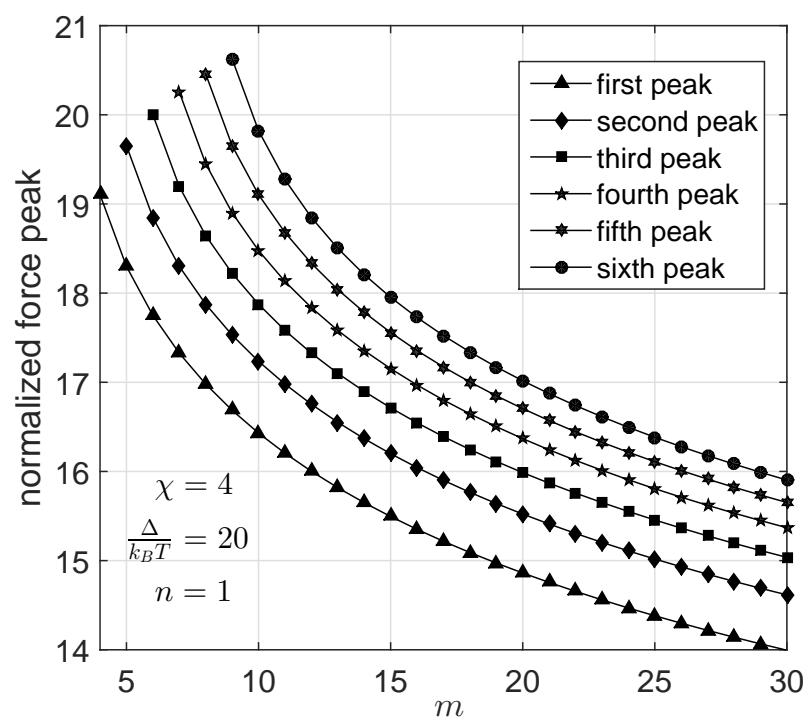

Fig. 8 Normalized force $\eta$, corresponding to the first six peaks of the force-extension response, versus the number of elements of the chain $m=4, \ldots, 30$ (triangles: first peak, diamonds: second peak, squares: third peak, pentagrams: fourth peak, hexagrams: fifth peak, and circles: sixth peak). We considered a one-dimensional system $(n=1)$ with $\chi=4$ and $\frac{\Delta}{k_{B} T}=20$. The force peaks decreasing with $m$ quantitatively describes the convergence to the thermodynamic limit.

\section{Conclusions}

We studied the force-extension response of lattice polymer models within the Gibbs (isotensional) and the Helmholtz (isometric) ensembles. By means of an exact representation of the Helmholtz partition function with a lattice Green function, and through its asymptotic development obtained through the multivariate saddle point method, we proved the equivalence of the ensembles in the thermodynamic limit, i.e. for very long, ideally infinite, chains. The possible deviation in the force-extension curves, for different ensembles, observable for rather short chain is a typical effect of the thermodynamic and of the statistical mechanics of small systems. These effects are important today not only from the theoretical point of view, but also from the experimental one, due to the recently introduced force spectroscopy techniques, which are able to probe the elasticity at molecular level. These concepts become even more meaningful when we consider polymers undergoing conformational transitions, i.e. composed of elements with two states, folded and unfolded. As a matter of fact, most of macromolecules of biological origin (proteins, nucleic acids and so on) tested with force spectroscopy techniques have shown this behavior. Therefore, we studied the isotensional and isometric responses of bistable or two-state lattice systems and we proved that the corresponding force-extension curves exhibit a plateau-like shape for the Gibbs ensemble and a sawtooth-like pattern for the Helmholtz one, as predicted by experiments. We finally rigorously proved that the ensembles equivalence in the thermodynamic limit is also valid for system with conformational transitions. We established that the lattice polymer models are able to reproduce all the peculiar features of the folding/unfolding process induced by mechanical stretching. To conclude, the models of folding/unfolding processes here presented can be also used to describe other situations with discrete phase transformations such as macromolecular hairpins, adhesive clusters, ferromagnetic alloys, indented substrates and plastic materials. 


\section{A The one-dimensional case}

If we take into consideration a one-dimensional system (without conformational transitions), the force-extension Gibbs response is simply given by

$$
\left\langle x_{m}\right\rangle=\ell m \tanh \left(\frac{f \ell}{k_{B} T}\right),
$$

as directly follows from Eq.(14) with $n=1$. The Helmholtz response in this simple case can be directly analysed, as presented below. We have $\sigma_{i}= \pm 1 \forall i=1, \ldots, m$ with the constraint $\sum_{i=1}^{m} \sigma_{i}=x \in \mathbb{Z}$. We have a total of $2^{m}$ trajectories and we need the number of trajectories satisfying the Helmholtz constraint. The total number of paths can be decomposed as $2^{m}=\sum_{k=0}^{m}\left(\begin{array}{c}m \\ k\end{array}\right)$, where $\left(\begin{array}{c}m \\ k\end{array}\right)$ represents the number of paths with $k$ times $\sigma_{i}=+1$ and $m-k$ times $\sigma_{i}=-1$. Now, we can write the Helmholtz condition as $\sum_{i=1}^{m} \sigma_{i}=x=a-b$, where $a$ is the number of $\sigma_{i}=+1$ and $b$ is the number of $\sigma_{i}=-1$. From the equations $a+b=m$ and $a-b=x$, we easily obtain $a=(m+x) / 2$ and $b=(m-x) / 2$. It follows that $\left(\begin{array}{c}m \\ a\end{array}\right)$ represents the number of paths with $a$ times $\sigma_{i}=+1$, i.e. the number of path arriving at $x$. The partition function is therefore given by

$$
Z_{H}(x)=\left(\begin{array}{c}
m \\
\frac{m+x}{2}
\end{array}\right)=\left(\begin{array}{c}
m \\
\frac{m-x}{2}
\end{array}\right)=\frac{m !}{\left(\frac{m+x}{2}\right) !\left(\frac{m-x}{2}\right) !},
$$

where $x=x_{m} / \ell$, being $x_{m}$ the physical extension of the chain. Of course, Eq.(61) perfectly agrees with Eq.(8) with $n=1$. In this simple case the force-extension response can be directly calculated by means of the Eq.(16) since the partition function in Eq.(61) can be extended to real values of $x$ by means of the gamma function. Indeed, we have

$$
\langle f\rangle=-k_{B} T \frac{\partial}{\partial x_{m}} \log Z_{H}\left(x_{m}\right)=-k_{B} T \frac{\partial}{\partial x_{m}} \log \frac{\Gamma(m+1)}{\Gamma\left(\frac{m}{2}+\frac{x_{m}}{2 \ell}+1\right) \Gamma\left(\frac{m}{2}-\frac{x_{m}}{2 \ell}+1\right)}
$$

The derivative of the gamma function can be calculated through the definition of the digamma function $\psi(x)=\frac{\mathrm{d}}{\mathrm{d} x} \log \Gamma(x)$. We eventually get

$$
\langle f\rangle=\frac{k_{B} T}{2 \ell}\left[\psi\left(\frac{m}{2}+\frac{x_{m}}{2 \ell}+1\right)-\psi\left(\frac{m}{2}-\frac{x_{m}}{2 \ell}+1\right)\right] .
$$

Since the digamma function has the asymptotic representation $\psi(x) \simeq \log (x)$ for $x \rightarrow \infty$, and $y=x_{m} /(m \ell)$ is the normalized extension, the thermodynamic limit of the Helmholtz response reads

$$
\left\langle\frac{f \ell}{k_{B} T}\right\rangle \simeq \frac{1}{2} \log \frac{\frac{m}{2}+\frac{m y}{2}+1}{\frac{m}{2}-\frac{m y}{2}+1} \simeq \frac{1}{2} \log \frac{1+y}{1-y}=\operatorname{artanh}(y) .
$$

It follows that, for $m \rightarrow \infty$, we can write for the Helmholtz response $y=\tanh \left(\left\langle\frac{f \ell}{k_{B} T}\right\rangle\right)$, which is perfectly coherent with the Gibbs response introduced in Eq.(60).

\section{B The two-dimensional case}

Concerning the two-dimensional case, the Gibbs force-extension response of a system without conformational transitions is given by Eq.(21), with $n=2$. The two components of this equation can be explicitly written as

$$
\begin{aligned}
& \left\langle y_{1}\right\rangle\left(\cosh \eta_{1}+\cosh \eta_{2}\right)=\sinh \eta_{1}, \\
& \left\langle y_{2}\right\rangle\left(\cosh \eta_{1}+\cosh \eta_{2}\right)=\sinh \eta_{2},
\end{aligned}
$$

where we used the normalized forces and extensions. We easily obtain $\left\langle y_{1}\right\rangle /\left\langle y_{2}\right\rangle=\sinh \eta 1 / \sinh \eta_{2}$ and therefore we have that $\cosh \eta_{2}=\sqrt{1+\left(\left\langle y_{2}\right\rangle /\left\langle y_{1}\right\rangle\right)^{2} \sinh ^{2} \eta_{1}}$. So doing, Eq.(63) gives an equation for $\eta_{1}$, which solution can be found as

$$
\eta_{1}=\operatorname{artanh} \frac{2\left\langle y_{1}\right\rangle}{1+\left\langle y_{1}\right\rangle^{2}-\left\langle y_{2}\right\rangle^{2}} .
$$

A similar calculation (or a permutation of the indices) leads to the second component of the normalized force

$$
\eta_{2}=\operatorname{artanh} \frac{2\left\langle y_{2}\right\rangle}{1+\left\langle y_{2}\right\rangle^{2}-\left\langle y_{1}\right\rangle^{2}}
$$


We now study the Helmholtz ensemble and we show that we obtain the same behavior in the thermodynamic limit. For two-dimensional system, we describe two techniques to determine the Helmholtz partition function, i.e. the number of paths going from $(0,0)$ to $\left(x_{1}, x_{2}\right) \in \mathbb{Z}^{2}$.

To introduce the first technique, we observe that a sequence of segments $\Sigma$ of the $\mathbb{Z}^{2}$ lattice can be written as $\left(\boldsymbol{\sigma}_{1}, \ldots, \boldsymbol{\sigma}_{m}\right)$ with $\sigma_{i} \in\left\{ \pm \mathbf{e}_{1}, \pm \mathbf{e}_{2}\right\}$. So, we have a total number of paths equal to $4^{m}$ starting from from $(0,0)$. By making use of the multinomial expansion rule, this total number of paths can be decomposed as

$$
4^{m}=\sum_{p_{1}+n_{1}+p_{2}+n_{2}=m} \frac{m !}{p_{1} ! n_{1} ! p_{2} ! n_{2} !},
$$

where $\frac{m !}{p_{1} ! n_{1} ! p_{2} ! n_{2} !}$ is the number of paths with $p_{1}$ times $\mathbf{e}_{1}, n_{1}$ times $-\mathbf{e}_{1}, p_{2}$ times $\mathbf{e}_{2}$, and $n_{2}$ times $-\mathbf{e}_{2}$. Now we search the number of paths going from $(0,0)$ to $\left(x_{1}, x_{2}\right)$. Then we have

$$
\begin{aligned}
p_{1}-n_{1} & =x_{1}, \\
p_{2}-n_{2} & =x_{2}, \\
p_{1}+n_{1}+p_{2}+n_{2} & =m=2\left(n_{1}+n_{2}\right)+x_{1}+x_{2},
\end{aligned}
$$

where $p_{1}$ is the number of $\mathbf{e}_{1}, n_{1}$ is the number of $-\mathbf{e}_{1}, p_{2}$ is the number of $\mathbf{e}_{2}$, and $n_{2}$ is the number of $-\mathbf{e}_{2}$. Therefore, we can affirm that the number of paths between $(0,0)$ to $\left(x_{1}, x_{2}\right)$, composed of $m$ segments, is given by

$$
\begin{aligned}
Z_{H}\left(x_{1}, x_{2}\right) & =\sum_{n_{1}+n_{2}=\frac{m-x_{1}-x_{2}}{2}} \frac{m !}{n_{1} !\left(n_{1}+x_{1}\right) ! n_{2} !\left(n_{2}+x_{2}\right) !} \\
& =\sum_{n_{1}=0}^{\frac{m-x_{1}-x_{2}}{2}} \frac{m !}{n_{1} !\left(n_{1}+x_{1}\right) !\left(\frac{m-x_{1}-x_{2}}{2}-n_{1}\right) !\left(\frac{m-x_{1}+x_{2}}{2}-n_{1}\right) !} \\
& =\left(\begin{array}{c}
m \\
\frac{m-x_{1}-x_{2}}{2}
\end{array}\right) \sum_{n_{1}=0}^{\frac{m-x_{1}-x_{2}}{2}}\left(\begin{array}{c}
\frac{m-x_{1}-x_{2}}{2} \\
n_{1}
\end{array}\right)\left(\begin{array}{c}
\frac{m-x_{1}-x_{2}}{2}+x_{1}+x_{2} \\
n_{1}+x_{1}
\end{array}\right) .
\end{aligned}
$$

If now use the property

$$
\sum_{k=0}^{n}\left(\begin{array}{l}
n \\
k
\end{array}\right)\left(\begin{array}{l}
n+\alpha \\
k+\beta
\end{array}\right)=\left(\begin{array}{c}
2 n+\alpha \\
n+\beta
\end{array}\right),
$$

we obtain the simple result

$$
Z_{H}\left(x_{1}, x_{2}\right)=\left(\begin{array}{c}
m \\
\frac{m-x_{1}-x_{2}}{2}
\end{array}\right)\left(\begin{array}{c}
m \\
\frac{m+x_{1}-x_{2}}{2}
\end{array}\right) .
$$

It is not difficult to prove that Eq.(73) perfectly corresponds to Eq.(8) with $n=2$.

The same result can be obtained with the following alternative, more elegant, method. We define a biunivocal correspondence between one two-dimensional path and a couple of one-dimensional paths, as follows

$$
\begin{array}{ccc}
2 \text { D path } \Leftrightarrow \text { (first } 1 \mathrm{D} \text { path })+ \text { (second } 1 \text { D path) } \\
\uparrow\left(p_{2}\right) & \uparrow & \uparrow \\
\rightarrow\left(p_{1}\right) & \uparrow & \downarrow \\
\leftarrow\left(n_{1}\right) & \downarrow & \uparrow \\
\downarrow\left(n_{2}\right) & \downarrow & \downarrow
\end{array}
$$

This rule can be summed up by saying that the vertical segments remain unchanged to generate both the one-dimensional paths, whereas the horizontal ones must be modified as follows: in the first one-dimensional path rightward arrows become upward and leftward ones become downward; on the other hand, in the second one-dimensional path rightward arrows become downward and leftward ones become upward. It is clear that this rule defines a bijection between the space of the two-dimensional paths and the space of the couples of one-dimensional paths (all composed by $m$ segments). Now, the two-dimensional paths start at $(0,0)$ and arrive at $\left(x_{1}, x_{2}\right)$. It means that $p_{2}-n_{2}=x_{2}$ and $p_{1}-n_{1}=x_{1}$. Hence, the first associated one-dimensional path starts at 0 and arrives at $p_{2}+p_{1}-n_{1}-n_{2}=x_{1}+x_{2}$ and the second associated one-dimensional path starts at 0 and arrives at $p_{2}+n_{1}-p_{1}-n_{2}=x_{1}-x_{2}$. We can use Eq.(61) of Appendix A to affirm that the total number 
of $1 \mathrm{D}$ path of the first type is $\left(\frac{m-x_{1}-x_{2}}{2}\right)$ and the total number of $1 \mathrm{D}$ paths of the second type is $\left(\frac{m+x_{1}-x_{2}}{2}\right)$. Since we introduced a biunivocal correspondence, the total number of $2 \mathrm{D}$ paths is exactly the product of the above obtained quantities $\left(\frac{m-x_{1}-x_{2}}{2}\right)$ and $\left(\frac{m+x_{1}-x_{2}}{2}\right)$, finally confirming Eq.(73).

The Helmholtz response can be calculated through Eq.(16) since the partition function in Eq.(73), as in Appendix A, can be extended to real values of $x_{1}$ and $x_{2}$ by means of the gamma function. By using the identities $x_{m 1}=\ell x_{1}$ and $x_{m 2}=\ell x_{2}$, we have

$$
\begin{aligned}
\left\langle f_{1}\right\rangle= & -k_{B} T \frac{\partial}{\partial x_{m 1}} \log Z_{H}\left(x_{m 1}, x_{m 2}\right) \\
= & -k_{B} T \frac{\partial}{\partial x_{m 1}} \log \left[\frac{\Gamma(m+1)}{\Gamma\left(\frac{m}{2}+\frac{x_{m 1}}{2 \ell}+\frac{x_{m 2}}{2 \ell}+1\right) \Gamma\left(\frac{m}{2}-\frac{x_{m 1}}{2 \ell}-\frac{x_{m 2}}{2 \ell}+1\right)}\right. \\
& \left.\times \frac{\Gamma(m+1)}{\Gamma\left(\frac{m}{2}+\frac{x_{m 1}}{2 \ell}-\frac{x_{m 2}}{2 \ell}+1\right) \Gamma\left(\frac{m}{2}-\frac{x_{m 1}}{2 \ell}+\frac{x_{m 2}}{2 \ell}+1\right)}\right] \\
= & \frac{k_{B} T}{2 \ell}\left[\psi\left(\frac{m}{2}+\frac{x_{m 1}}{2 \ell}+\frac{x_{m 2}}{2 \ell}+1\right)-\psi\left(\frac{m}{2}-\frac{x_{m 1}}{2 \ell}-\frac{x_{m 2}}{2 \ell}+1\right)\right. \\
& \left.+\psi\left(\frac{m}{2}+\frac{x_{m 1}}{2 \ell}-\frac{x_{m 2}}{2 \ell}+1\right)-\psi\left(\frac{m}{2}-\frac{x_{m 1}}{2 \ell}+\frac{x_{m 2}}{2 \ell}+1\right)\right] .
\end{aligned}
$$

In terms of normalized force and extension, we finally get

$$
\begin{aligned}
\left\langle\eta_{1}\right\rangle= & \frac{1}{2}\left[\psi\left(\frac{m}{2}+\frac{m y_{1}}{2}+\frac{m y_{2}}{2}+1\right)-\psi\left(\frac{m}{2}-\frac{m y_{1}}{2}-\frac{m y_{2}}{2}+1\right)\right. \\
& \left.+\psi\left(\frac{m}{2}+\frac{m y_{1}}{2}-\frac{m y_{2}}{2}+1\right)-\psi\left(\frac{m}{2}-\frac{m y_{1}}{2}+\frac{m y_{2}}{2}+1\right)\right] .
\end{aligned}
$$

Since $\psi(x) \simeq \log (x)$ for $x \rightarrow \infty$, we obtain the asymptotic representation

$$
\left\langle\eta_{1}\right\rangle=\frac{1}{2} \log \frac{\left(1+y_{1}+y_{2}\right)\left(1+y_{1}-y_{2}\right)}{\left(1-y_{1}-y_{2}\right)\left(1-y_{1}+y_{2}\right)},
$$

in the limit of $m \rightarrow \infty$. If we finally consider the equation

$$
\frac{\left(1+y_{1}+y_{2}\right)\left(1+y_{1}-y_{2}\right)}{\left(1-y_{1}-y_{2}\right)\left(1-y_{1}+y_{2}\right)}=\frac{1+r}{1-r}
$$

we obtain that $r=\frac{2 y_{1}^{2}}{1+y_{1}^{2}-y_{2}^{2}}$ and, therefore, we have the result

$$
\left\langle\eta_{1}\right\rangle=\frac{1}{2} \log \frac{1+r}{1-r}=\operatorname{artanh} r=\operatorname{artanh} \frac{2 y_{1}^{2}}{1+y_{1}^{2}-y_{2}^{2}},
$$

which is in perfect agreement with Eq.(65). The same result can be easily proved for $\left\langle\eta_{2}\right\rangle$.

\section{The three-dimensional case}

For the three-dimensional case, the Gibbs force-extension response is given by Eq.(21), with $n=3$. The three components of this equation can be explicitly written as follows

$$
\begin{aligned}
& \left\langle y_{1}\right\rangle\left(\cosh \eta_{1}+\cosh \eta_{2}+\cosh \eta_{3}\right)=\sinh \eta_{1}, \\
& \left\langle y_{2}\right\rangle\left(\cosh \eta_{1}+\cosh \eta_{2}+\cosh \eta_{3}\right)=\sinh \eta_{2}, \\
& \left\langle y_{3}\right\rangle\left(\cosh \eta_{1}+\cosh \eta_{2}+\cosh \eta_{3}\right)=\sinh \eta_{3} .
\end{aligned}
$$

In this case the generalized force $\boldsymbol{\eta}$ cannot be explicitly written in terms of the generalized extension $\langle\mathbf{y}\rangle$. However, if we consider $\eta_{1}=\eta_{2}=0$ and $\eta_{3} \triangleq \eta \neq 0$ (uni-axial stretching), we have that $\left\langle y_{1}\right\rangle=\left\langle y_{2}\right\rangle=0$ and $\left\langle y_{3}\right\rangle \triangleq\langle y\rangle \neq 0$. In these conditions, by means of a long but straightforward calculation, we get

$$
\eta=\log \left(\frac{2\langle y\rangle+\langle y\rangle \sqrt{1+3\langle y\rangle^{2}}+\sqrt{1+3\langle y\rangle^{2}+4\langle y\rangle^{2} \sqrt{1+3\langle y\rangle^{2}}+4\langle y\rangle^{4}}}{1-\langle y\rangle^{2}}\right) .
$$


To analyse the Helmholtz ensemble, we remark that a sequence of segments $\Sigma$ of the $\mathbb{Z}^{3}$ lattice can be written as $\left(\boldsymbol{\sigma}_{1}, \ldots, \boldsymbol{\sigma}_{m}\right)$ with $\boldsymbol{\sigma}_{i} \in\left\{ \pm \mathbf{e}_{1}, \pm \mathbf{e}_{2}, \pm \mathbf{e}_{3}\right\}$. So, we have a total number of paths equal to $6^{m}$ starting from from $(0,0)$. By making use of the multinomial expansion rule, this total number of paths can be decomposed as

$$
6^{m}=\sum_{p_{1}+n_{1}+p_{2}+n_{2}+p_{3}+n_{3}=m} \frac{m !}{p_{1} ! n_{1} ! p_{2} ! n_{2} ! p_{3} ! n_{3} !}
$$

where $\frac{m !}{p_{1} ! n_{1} ! p_{2} ! n_{2} ! p_{3} ! n_{3} !}$ is the number of paths with $p_{1}$ times $\mathbf{e}_{1}, n_{1}$ times $-\mathbf{e}_{1}, p_{2}$ times $\mathbf{e}_{2}, n_{2}$ times $-\mathbf{e}_{2}, p_{3}$ times $\mathbf{e}_{3}$, and $n_{3}$ times $-\mathbf{e}_{3}$. Now we search the number of paths going from $(0,0,0)$ to $\left(x_{1}, x_{2}, x_{3}\right)$. Then we have

$$
\begin{aligned}
p_{1}-n_{1} & =x_{1}, \\
& p_{2}-n_{2}=x_{2}, \\
& p_{3}-n_{3}=x_{3}, \\
p_{1}+n_{1}+p_{2}+n_{2}+ & p_{3}+n_{3}=m=2\left(n_{1}+n_{2}+n_{3}\right)+x_{1}+x_{2}+x_{3} .
\end{aligned}
$$

Therefore, we can determine the number of paths between $(0,0,0)$ to $\left(x_{1}, x_{2}, x_{3}\right)$, composed of $m$ segments, as follows

$$
\begin{aligned}
Z_{H}\left(x_{1}, x_{2}, x_{3}\right) & =\sum_{n_{1}+n_{2}+n_{3}=\frac{m-x_{1}-x_{2}-x_{3}}{2}} \frac{m !}{n_{1} !\left(n_{1}+x_{1}\right) ! n_{2} !\left(n_{2}+x_{2}\right) ! n_{3} !\left(n_{3}+x_{3}\right) !} \\
& =\sum_{n_{1}=0}^{M} \sum_{n_{2}=0}^{M-n_{1}} \frac{m !}{n_{1} !\left(n_{1}+x_{1}\right) ! n_{2} !\left(n_{2}+x_{2}\right) !\left(M-n_{1}-n_{2}\right) !\left(M-n_{1}-n_{2}+x_{3}\right) !},
\end{aligned}
$$

where we introduced $M=\frac{m-x_{1}-x_{2}-x_{3}}{2}$ to simplify the notation. It is now possible to identify the binomial coefficients to simplify the expression of the Helmholtz partition function

$$
\begin{aligned}
Z_{H}\left(x_{1}, x_{2}, x_{3}\right) & =\left(\begin{array}{c}
m \\
M
\end{array}\right) \sum_{n_{1}=0}^{M} \sum_{n_{2}=0}^{M-n_{1}}\left(\begin{array}{c}
M \\
n_{1}
\end{array}\right)\left(\begin{array}{c}
M-n_{1} \\
n_{2}
\end{array}\right)\left(\begin{array}{c}
M+x_{1}+x_{2}+x_{3} \\
n_{1}+x_{1}
\end{array}\right)\left(\begin{array}{c}
M+x_{2}+x_{3}-n_{1} \\
n_{2}+x_{2}
\end{array}\right) \\
& =\left(\begin{array}{c}
m \\
\frac{m-x_{1}-x_{2}-x_{3}}{2}
\end{array}\right) \sum_{n_{1}=0}^{\frac{m-x_{1}-x_{2}-x_{3}}{2}}\left(\begin{array}{c}
\frac{m-x_{1}-x_{2}-x_{3}}{2} \\
n_{1}
\end{array}\right)\left(\begin{array}{c}
\frac{m+x_{1}+x_{2}+x_{3}}{2} \\
n_{1}+x_{1}
\end{array}\right)\left(\begin{array}{c}
m-x_{1}-2 n_{1} \\
\frac{m-x_{1}+x_{2}-x_{3}}{2}-n_{1}
\end{array}\right),
\end{aligned}
$$

where we used Eq.(72) to evaluate one of the two sums. Unfortunately, the last sum in Eq.(87) cannot be calculated explicitly, leaving the final result in a more complicated form than that obtained for $n=1$ or $n=2$ (see Appendices A and B). In any case, Eq.(87) can be proved to be in perfect agreement with the general result stated in Eq.(8), applied with $n=3$. When we consider a uni-axial stretching, as in Eq.(80), the expression of the Helmholtz partition function, given in Eq.(87), can be simplified as follows

$$
Z_{H}(x)=\left(\begin{array}{c}
m \\
\frac{m-x}{2}
\end{array}\right) \sum_{n=0}^{\frac{m-x}{2}}\left(\begin{array}{c}
\frac{m-x}{2} \\
n
\end{array}\right)\left(\begin{array}{c}
\frac{m+x}{2} \\
n
\end{array}\right)\left(\begin{array}{c}
m-2 n \\
\frac{m-x}{2}-n
\end{array}\right),
$$

where we considered $x_{1}=0, x_{2}=0$ and $x_{3}=x$. The force-extension relation can be finally written in the following form

$$
\langle\eta\rangle=\frac{1}{2} \log \frac{Z_{H}(m y-1)}{Z_{H}(m y+1)}
$$

and it can be compared with Eq.(80).

In Appendix A and B we have readily proved the equivalence of the ensembles for one- and two-dimensional lattice polymers, since in this cases the Helmholtz partition functions can be extended to real values of the arguments without difficulties, see Eqs.(61) and (73), and the derivatives can be directly calculated. In the three-dimensional case (or in spaces of higher dimension), this procedure cannot be followed in consideration of the sum which appears in the partition function expression, see Eq.(87) or Eq.(88). Indeed, since the upper limit of the sum depends on $\left(x_{1}, x_{2}, x_{3}\right)$ in Eq.(87) or on $x$ in Eq.(88), it is not possible to extend the validity of these expressions to real arguments and we cannot apply any differential operator. The conclusion is that for proving the ensemble equivalence we must follow the general procedure introduced in the main text (see Section 5). However, the Gibbs response given in Eq.(80) and the Helmholtz one stated in Eqs.(88) and (89) are useful to compare the two behaviors and to graphically observe the convergence towards the thermodynamic limit, as shown in Fig.3 of Section 5. 


\section{References}

1. J. H. Weiner, Statistical Mechanics of Elasticity, Dover Publication Inc., New York (2002).

2. M. Doi, Introduction to Polymer Physics, Clarendon, Oxford (1996).

3. C. Vanderzande, Lattice Models of Polymers, Cambridge University Press, Cambridge (1998).

4. E. J. Janse van Rensburg, Statistical Mechanics of Interacting Walks, Polygons, Animals and Vesicles, Oxford University Press Inc., New York (2000).

5. P. J. Flory, Statistical Mechanics of Polymer Chains, John Wiley and Sons, New York (1989).

6. M. Rubinstein, R.H. Colby, Polymer Physics, Oxford University Press, New York (2003).

7. K. Binder (Editor), The Monte Carlo Method in Condensed Matter Physics, Topics in Applied Physics, Vol. 71, Springer-Verlag, Berlin, Heidelberg (1992).

8. K. Binder, D. Heermann, Monte Carlo Simulation in Statistical Physics: An Introduction, Springer-Verlag, Berlin, Heidelberg (2010).

9. Halpin-Healy T., Zhang Y.C., Kinetic roughening phenomena, stochastic growth, directed polymers and all that. Aspects of multidisciplinary statistical mechanics, Phys. Rep. 254, 215 (1995).

10. P. G. de Gennes, Scaling concepts in polymer physics, Cornell University Press, Ithaca (1979).

11. P. G. de Gennes, Exponents for the excluded volume problem as derived by the Wilson method, Phys. Lett. A 38, 339 (1972).

12. T. R. Strick, M.-N. Dessinges, G. Charvin, N. H. Dekker, J.-F. Allemand, D. Bensimon and V. Croquette, Stretching of macromolecules and proteins, Rep. Progr. Phys. 66, 1 (2002).

13. K. C. Neuman, A. Nagy, Single-molecule force spectroscopy: optical tweezers, magnetic tweezers and atomic force microscopy, Nature Meth. 5, 491 (2008).

14. S. Kumar, M. S. Li, Biomolecules under mechanical force, Physics Reports 486, 1 (2010).

15. C. Bustamante, J. Liphardt and F. Ritort, The Nonequilibrium Thermodynamics of Small Systems, Physics Today 58, 43 (2005).

16. E. Dieterich, J. Camunas-Soler, M. Ribezzi-Crivellari, U. Seifert, and F. Ritort, Control of force through feedback in small driven systems, Phys. Rev. E 94, 012107 (2016).

17. R.G. Winkler, Deformation of semiflexible chains, J. Chem. Phys. 118, 2919 (2003).

18. J. F. Marko, E. D. Siggia, Stretching DNA, Macromolecules 28, 8759 (1995).

19. G. Glatting, R.G. Winkler, P. Reineker, Partition function and force extension relation for a generalized freely jointed chain, Macromolecules 26, 6085 (1993).

20. F. Manca, S. Giordano, P. L. Palla, R. Zucca, F. Cleri, and L. Colombo, Elasticity of flexible and semiflexible polymers with extensible bonds in the Gibbs and Helmholtz ensembles, J. Chem. Phys., 136, 154906 (2012).

21. F. Manca, S. Giordano, P. L. Palla, F. Cleri, and L. Colombo, Response to Comment on Elasticity of flexible and semiflexible polymers with extensible bonds in the Gibbs and Helmholtz ensembles, J. Chem. Phys., 138, 157102 (2013).

22. Manca, F., Giordano, S., Palla, P.L., Cleri, F., Colombo, L., Theory and Monte Carlo simulations for the stretching of flexible and semiflexible single polymer chains under external fields, J. Chem. Phys. 137, 244907 (2012).

23. A. Rosa, T. X. Hoang, D. Marenduzzo, and A. Maritan, A new interpolation formula for semiflexible polymers, Biophys. Chem. 115, 251 (2005).

24. T. Su and P. K. Purohit, Thermomechanics of a heterogeneous fluctuating chain, J. Mech. Phys. Solids $58,164(2010)$.

25. J. Kierfeld, O. Niamploy, V. Sa-Yakanit, and R. Lipowsky, Stretching of semiflexible polymers with elastic bonds, Eur. Phys. J. E 14, 17 (2004).

26. R. G. Winkler, Equivalence of statistical ensembles in stretching single flexible polymers, Soft Matter 6 , $6183(2010)$.

27. F. Manca, S. Giordano, P. L. Palla, F. Cleri, On the equivalence of thermodynamics ensembles for flexible polymer chains, Phys. A Stat. Mech. its Appl. 395, 154 (2014).

28. M. Rief, J. M. Fernandez, H. E. Gaub, Elastically Coupled Two-Level Systems as a Model for Biopolymer Extensibility, Phys. Rev. Lett. 81, 4764 (1998).

29. F. Manca, S. Giordano, P. L. Palla, F. Cleri, L. Colombo, Two-state theory of single-molecule stretching experiments, Phys. Rev. E 87, 032705 (2013).

30. M. S. Kellermayer, S. B. Smith, H. L. Granzier, and C. Bustamante, Folding-unfolding transitions in single titin molecules characterized with laser tweezers, Science 276, 1112 (1997).

31. M. Rief, J. Pascual, M. Saraste, and H. E. Gaub, Single molecule force spectroscopy of spectrin repeats: low unfolding forces in helix bundles, J. Mol. Biol. 286, 553 (1999).

32. A. Prados, A. Carpio, and L. L. Bonilla, Sawtooth patterns in force-extension curves of biomolecules: An equilibrium-statistical-mechanics theory, Phys. Rev. E 88, 012704 (2013).

33. L. L. Bonilla, A. Carpio, and A. Prados, Theory of force-extension curves for modular proteins and DNA hairpins, Phys. Rev. E 91, 052712 (2015).

34. S. Giordano, Spin variable approach for the statistical mechanics of folding and unfolding chains, Soft Matter 13, 6877 (2017).

35. M. Caruel, J.-M. Allain, L. Truskinovsky, Mechanics of collective unfolding, J. Mech. Phys. Sol. 76, 237 (2015).

36. I. Benichou, Y. Zhang, O. K. Dudko, S. Givli, The rate dependent response of a bistable chain at finite temperature, J. Mech. Phys. Sol. 95, 44 (2016).

37. E. Parzen, Modern Probability Theory and Its Applications, Wiley-Interscience, New York (1992).

38. A. V. Oppenheim, R. W. Schafer, Discrete-Time Signal Processing, Prentice Hall, Upper Saddle River, New Jersey (1999). 
39. T. H. Berlin and M. Kac, The spherical model of a ferromagnet, Phys. Rev. 86, 821 (1952).

40. T. Morita, T. Horiguchi, Calculation of the Lattice Green's Function for the bcc, fcc, and Rectangular Lattices, J. Math. Phys. 12, 986 (1971).

41. R. T. Delves, G. S. Joyce, On the Green Function for the Anisotropic Simple Cubic Lattice, Annals of Physics 291, 71 (2001).

42. J. Bernasconi, Conduction in anisotropic disordered systems: effective-medium theory. Phys. Rev. B 9, 4575 (1974).

43. J. Cserti, Application of the lattice Green's function for calculating the resistance of an infinite network of resistors, Am. J. Phys. 68, 896 (2000).

44. S. Giordano, Disordered lattice networks: general theory and simulations, Int. J. Circ. Theor. Appl. 33, $519(2005)$

45. S. Giordano, Two-dimensional disordered lattice networks with substrate, Physica A: Statistical Mechanics and its Applications 375, 726 (2007).

46. Q. Wang, On the anisotropy of lattice polymers, J. Chem. Phys. 131, 234903 (2009).

47. G. Glatting, R. G. Winkler, P. Reineker, Are the continuum and the lattice representation of freely jointed chains equivalent?, Macromol. Theory Simul. 3, 575 (1994).

48. M. P. Taylor, J. E. G. Lipson, Lattice versus continuum models of a polymer chain, J. Chem. Phys. 111, 8701 (1999).

49. M. V. Fedoryuk, Asymptotic: Integrals and Series, Nauka, Moscow (1987).

50. R. Wong, Asymptotic approximations of integrals, Academic Press, New York (1989).

51. R. Pemantle, M. C. Wilson, Asymptotic expansions of oscillatory integrals with complex phase, Contemporary Mathematics 520, 221 (2010).

52. T. Neuschel, Apéry Polynomials and the Multivariate Saddle Point Method, Constructive Approximation 40, $487(2014)$

53. S. M. Smith, Y. Cui, C. Bustamante, Overstretching B-DNA: the elastic response of individual doublestranded and single-stranded DNA molecules, Science 271, 795 (1996).

54. C. Bustamante, Z. Bryant, S. B. Smith, Ten years of tension: single-molecule DNA mechanics, Nature 421 , $423(2003)$

55. I. Rouzina, V. A. Bloomfield, Force-induced melting of the DNA double helix 1. Thermodynamic analysis, Biophys. J. 80, 882 (2001).

56. I. Rouzina, V. A. Bloomfield, Force-induced melting of the DNA double helix 2. Effect of solution conditions., Biophys. J. 80, 894 (2001).

57. A. E. B. Pupo, F. Falo, A. Fiasconaro, DNA overstretching transition induced by melting in a dynamical mesoscopic model, J. Chem. Phys. 139, 095101 (2013).

58. S. Cocco, J. Yan, J.-F. Léger, D. Chatenay, J. F. Marko, Overstretching and force-driven strand separation of double-helix DNA, Phys. Rev. E 70, 011910 (2004).

59. M. Rief, F. Oesterhelt, B. Heymann, H. E. Gaub, Single Molecule Force Spectroscopy on Polysaccharides by Atomic Force Microscopy, Science 275, 28 (1997).

60. F. Hanke and H. J. Kreuzer, Conformational transitions in single polymer molecules modeled with a complete energy landscape: continuous two-state model, Eur. Phys. J. E 22, 163 (2007).

61. G. I. Bell, Models for the specific adhesion of cells to cells, Science 200, 618 (1978).

62. T. E. Fisher, A. F. Oberhauser, M. Carrion-Vazquez, P. E. Marszalek, J. M. Fernandez, The study of protein mechanics with the atomic force microscope, Trends Biochem. Sci. 24, 379 (1999).

63. A. Imparato, F. Sbrana, M. Vassalli, Reconstructing the free-energy landscape of a polyprotein by singlemolecule experiments, Europhys Lett. 82, 58006 (2008).

64. D. De Tommasi, N. Millardi, G. Puglisi, G. Saccoamandi, An energetic model for macromolecules unfolding in stretching experiments, J. R. Soc. Interface 10, 20130651 (2013)

65. D. E. Makarov, A Theoretical Model for the Mechanical Unfolding of Repeat Proteins, Biophys. J. 96, 2160 (2009).

66. G. Puglisi, L. Truskinovsky, Mechanics of a discrete chain with bi-stable elements, J. Mech. Phys. Sol. 48, 1 (2000).

67. M. Caruel, J.-M. Allain, L. Truskinovsky, Muscle as a Metamaterial Operating Near a Critical Point, Phys. Rev. Lett. 110, 248103 (2013).

68. M. Caruel, L. Truskinovsky, Statistical mechanics of the Huxley-Simmons model, Phys. Rev. E 93, 062407 (2016).

69. Y. R. Efendiev, L. Truskinovsky, Thermalization of a driven bi-stable FPU chain, Continuum Mech. Thermodyn. 22, 679 (2010)

70. I. Benichou, S. Givli, Structures undergoing discrete phase transformation, J. Mech. Phys. Sol. 61, 94 (2013).

71. I. Benichou, S. Givli, Rate Dependent Response of Nanoscale Structures Having a Multiwell Energy Landscape, Phys. Rev. Lett. 114, 095504 (2015). 